Supporting Information for:

\title{
Application of Tautomerism of Ferrocenyl Secondary Phosphine Oxides in Suzuki-Miyaura Cross Coupling Reactions
}

\author{
Lin-Ying Jung, Shih-Hung Tsai and Fung-E Hong* \\ Department of Chemistry, National Chung Hsing University, \\ 250 Kuo-Kuang Road, Taichung 40227, Taiwan \\ FAX: 886-4-22862547 \\ E-mail: fehong@dragon.nchu.edu.tw
}

*Author to whom correspondence should be addressed. 


\section{Contents}

* Fig. 1 ORTEP drawing of 7aB.

* Fig. 2 ORTEP drawing of 7aM.

* Fig. 3 ORTEP drawing of $7 \mathbf{b M}$.

* Fig. 4 ORTEP drawing of 8 .

* Fig. 5 ORTEP drawing of 9.

* Table 1. Crystal data of 7aB, 7aM, 7bM, 8 and 9

* Spectroscopic data for $7 \mathbf{a B}$

Elemental analysis for $\mathbf{7 a B}$

MS for $\mathbf{7 a B}$

${ }^{1} \mathrm{H}$ NMR for $\mathbf{7 a B}$

${ }^{13} \mathrm{C}$ NMR for $7 \mathbf{a B}$

${ }^{31} \mathrm{P}$ NMR for $\mathbf{7 a B}$

* Spectroscopic data for 7aM

Elemental analysis for $\mathbf{7 a M}$

MS for 7aM

${ }^{1} \mathrm{H}$ NMR for $\mathbf{7 a M}$

${ }^{13} \mathrm{C}$ NMR for $7 \mathbf{a M}$

${ }^{31} \mathrm{P}$ NMR for $7 \mathbf{a M}$

* $\quad$ Spectroscopic data for $7 \mathbf{b M}$

Elemental analysis for $\mathbf{7 b M}$

MS for 7bM

${ }^{1} \mathrm{H}$ NMR for $\mathbf{7 b M}$

${ }^{13} \mathrm{C}$ NMR for $7 \mathrm{bM}$

${ }^{31} \mathrm{P}$ NMR for $\mathbf{7 b M}$

* $\quad$ Spectroscopic data for $\mathbf{8}$

Elemental analysis for $\mathbf{8}$

MS for 8

${ }^{1} \mathrm{H}$ NMR for 8

${ }^{13} \mathrm{C}$ NMR for 8

${ }^{31} \mathrm{P}$ NMR for 8

* Spectroscopic data for 9

Elemental analysis for 9

MS for 9

${ }^{1} \mathrm{H}$ NMR for 9

${ }^{13} \mathrm{C}$ NMR for 9

${ }^{31} \mathrm{P}$ NMR for 9 


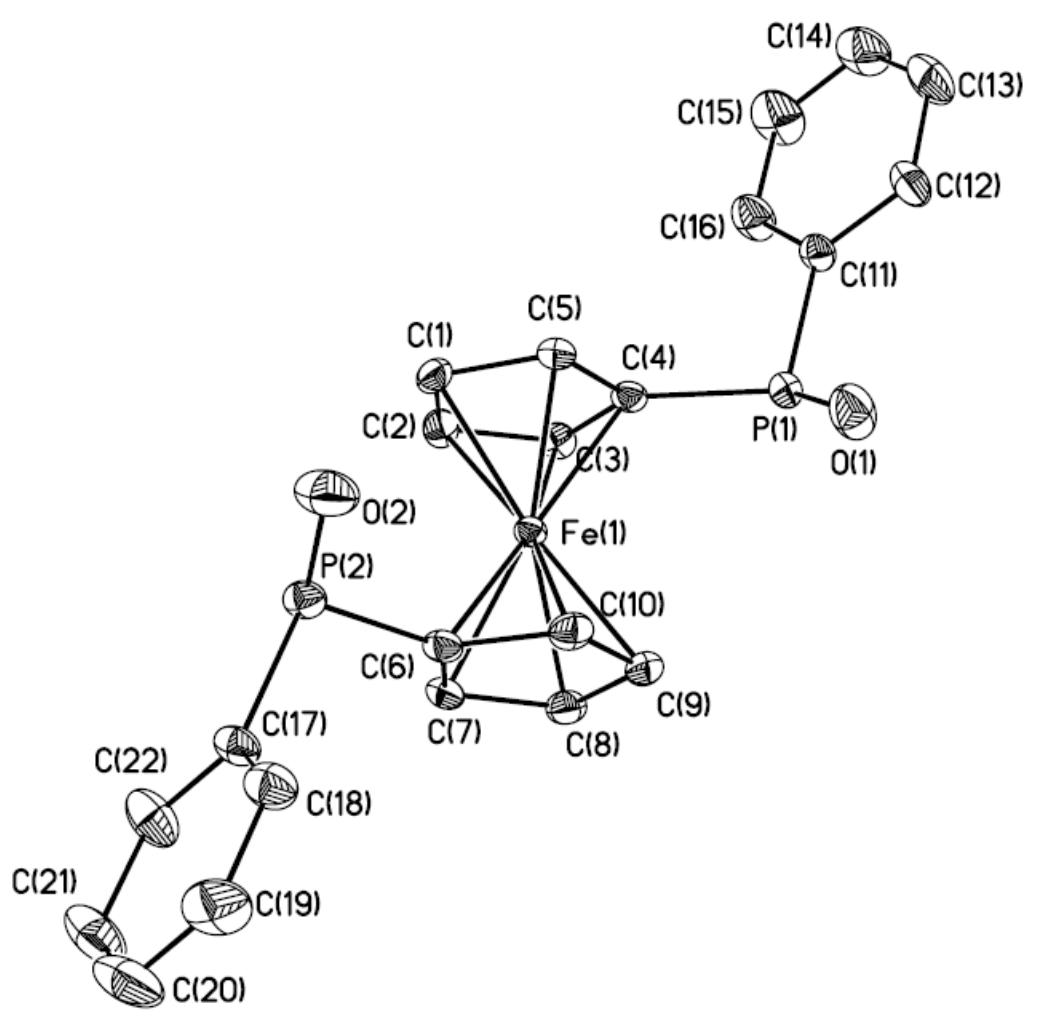

Fig. 1 ORTEP drawing of 7aB. Hydrogen atoms are omitted for clarity. Selected bond lengths $(\AA)$ and angles $\left({ }^{\circ}\right)$ : $\mathrm{Fe}(1)-\mathrm{C}(4)$ 2.011(5); $\mathrm{Fe}(1)-\mathrm{C}(6)$ 2.036(5); $\mathrm{P}(1)-\mathrm{O}(1)$ 1.504(5); $\mathrm{P}(1)-\mathrm{C}(4)$ 1.788(5); $\mathrm{P}(1)-\mathrm{C}(11)$ 1.794(6); $\mathrm{P}(2)-\mathrm{O}(2)$ 1.491(5); $\mathrm{P}(2)-\mathrm{C}(6)$ 1.784(5); $\mathrm{P}(2)-\mathrm{C}(17)$ 1.809(5); O(1)-P(1)-C(4) 114.0(3); O(1)-P(1)-C(11) 112.8(3); $\mathrm{C}(4)-\mathrm{P}(1)-\mathrm{C}(11)$ 106.0(3); O(2)-P(2)-C(6) 113.4(3); O(2)-P(2)-C(17) 112.9(3); $\mathrm{C}(6)-\mathrm{P}(2)-\mathrm{C}(17)$ 106.2(3); C(3)-C(4)-P(1) 125.6(4); C(5)-C(4)-P(1) 125.7(4); $\mathrm{C}(7)-\mathrm{C}(6)-\mathrm{P}(2)$ 126.4(4); C(10)-C(6)-P(2) 125.5(4). 


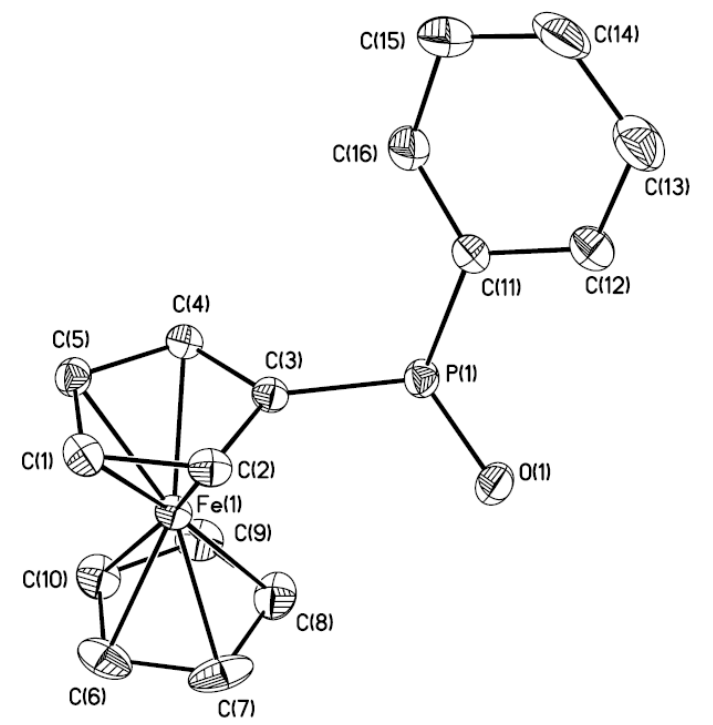

Fig. 2 ORTEP drawing of 7aM. Hydrogen atoms are omitted for clarity. Selected bond lengths $(\AA)$ and angles $\left(^{\circ}\right)$ : $\mathrm{Fe}(1)-\mathrm{C}(3)$ 2.026(2); $\mathrm{P}(1)-\mathrm{O}(1)$ 1.4792(19); $\mathrm{P}(1)-\mathrm{C}(3)$ 1.774(2); $\mathrm{P}(1)-\mathrm{C}(11)$ 1.805(3); $\mathrm{P}(1)-\mathrm{H}(1 \mathrm{~B})$ 1.3482; $\mathrm{O}(1)-\mathrm{P}(1)-\mathrm{C}(3)$ 116.64(12); $\mathrm{O}(1)-\mathrm{P}(1)-\mathrm{C}(11)$ 112.91(12); C(3)-P(1)-C(11) 106.31(11); O(1)-P(1)-H(1B) 115.0; $\mathrm{C}(3)-\mathrm{P}(1)-\mathrm{H}(1 \mathrm{~B}) 102.3$; C(11)-P(1)-H(1B) 102.1; C(2)-C(3)-P(1) 126.22(19); $\mathrm{C}(4)-\mathrm{C}(3)-\mathrm{P}(1)$ 126.43(18); $\mathrm{P}(1)-\mathrm{C}(3)-\mathrm{Fe}(1)$ 125.73(12).

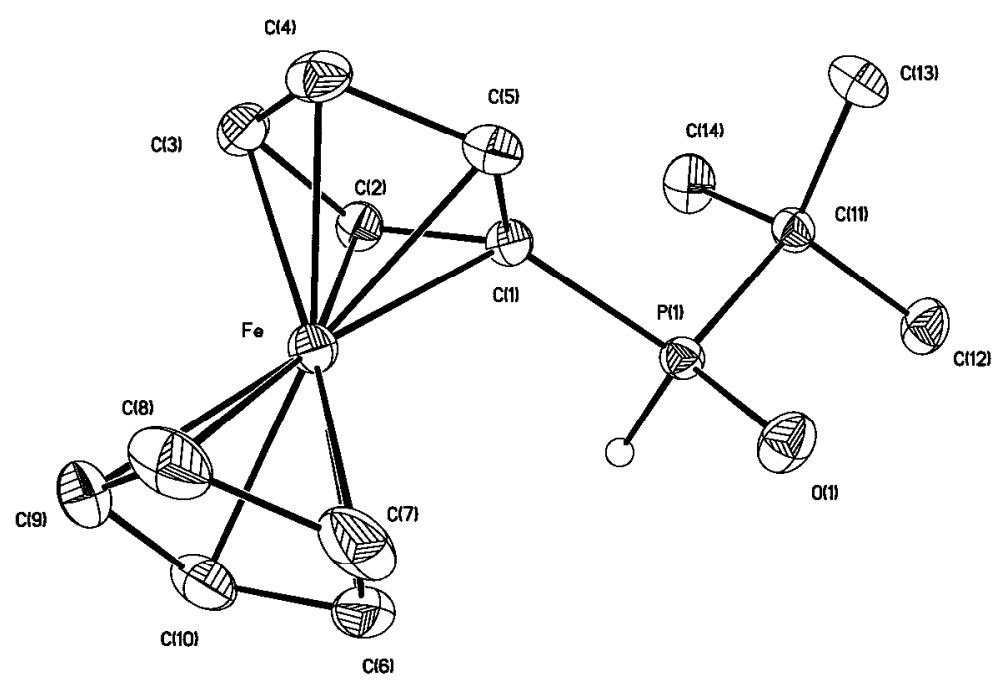

Fig. 3 ORTEP drawing of 7bM. Hydrogen atoms are omitted for clarity. Selected bond lengths $(\AA)$ and angles $\left({ }^{\circ}\right)$ : Fe-C(1) 2.030(2); Fe-C(2) 2.058(3); Fe-C(3) 2.053(3); Fe-C(4) 2.049(3); Fe-C(5) 2.030(3); P(1)-O(1) 1.484(2); P(1)-C(1) 1.788(3); $\mathrm{P}(1)-\mathrm{C}(11)$ 1.824(3); P(1)-H 1.32(3); C(2)-C(1) 1.429(4); C(2)-C(3) 1.423(4); $\mathrm{C}(3)-\mathrm{C}(4)$ 1.406(5); C(4)-C(5) 1.411(5); C(5)-C(1) 1.423(4); O(1)-P(1)-C(1) 113.80(13); O(1)-P(1)-C(11) 113.69(13); C(1)-P(1)-C(11) 107.77(13); O(1)-P(1)-H 115.3(13); C(1)-P(1)-H 104.2(13); C(11)-P(1)-H 100.9(13). 


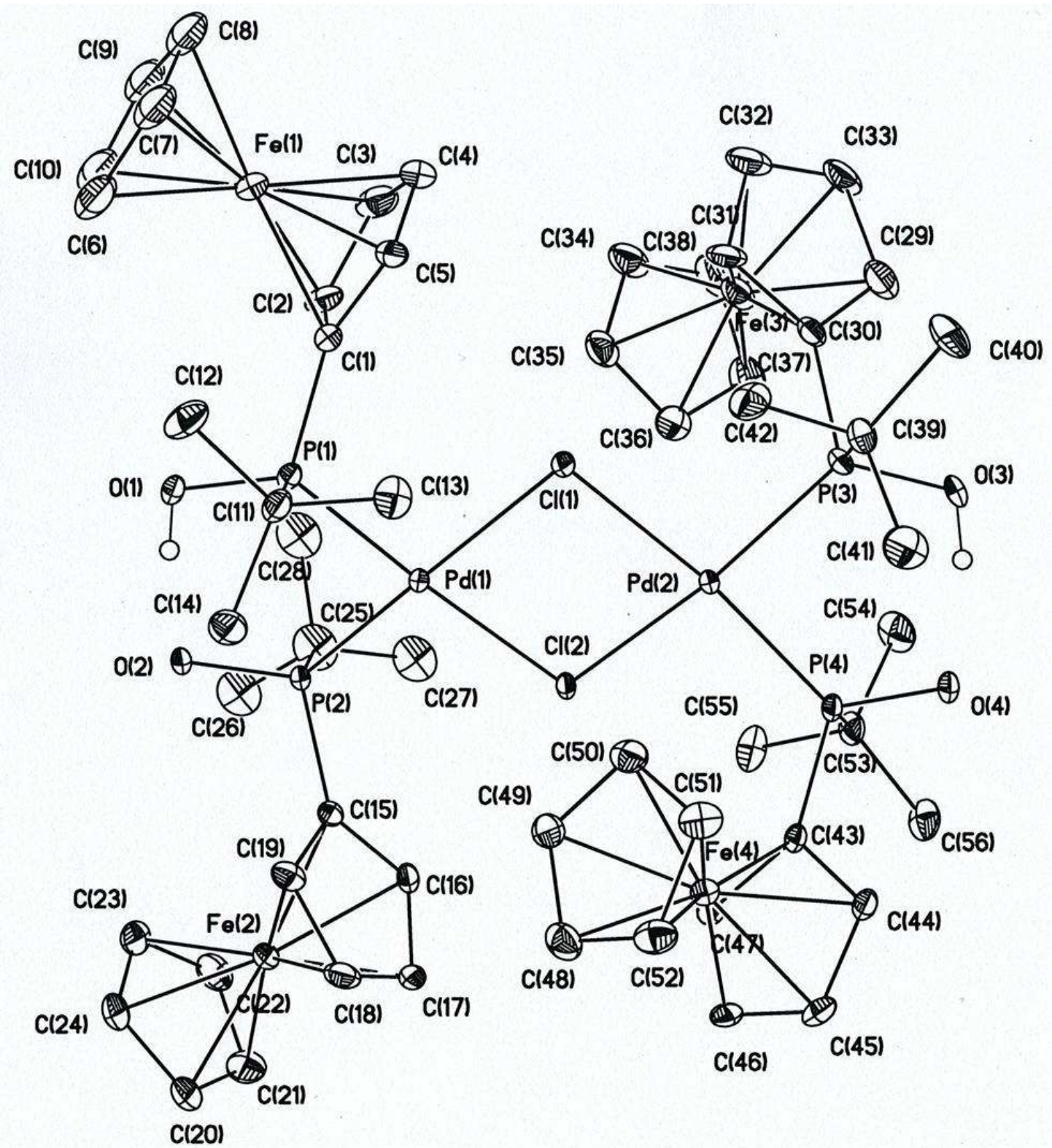

Fig. 4 ORTEP drawing of $\mathbf{8}$. Hydrogen atoms are omitted for clarity. Selected bond lengths $(\AA)$ and angles $\left({ }^{\circ}\right): \mathrm{Pd}(1)-\mathrm{P}(1)$ 2.248(3); $\mathrm{Pd}(1)-\mathrm{P}(2)$ 2.252(2); $\mathrm{P}(1)-\mathrm{O}(1)$ 1.557(7); $\mathrm{P}(1)-\mathrm{C}(1)$ 1.787(12); $\mathrm{P}(1)-\mathrm{C}(11)$ 1.836(13); $\mathrm{P}(1)-\mathrm{Pd}(1)-\mathrm{P}(2)$ 92.57(9); $\mathrm{Cl}(1)-\mathrm{Pd}(1)-\mathrm{Cl}(2)$ 82.66(9); $\mathrm{Pd}(2)-\mathrm{Cl}(1)-\mathrm{Pd}(1)$ 97.23(9); O(1)-P(1)-C(1) 106.1(5); $\mathrm{O}(1)-\mathrm{P}(1)-\mathrm{C}(11)$ 106.0(5); C(1)-P(1)-C(11) 112.8(6); O(1)-P(1)-Pd(1) 117.1(3); $\mathrm{C}(1)-\mathrm{P}(1)-\mathrm{Pd}(1)$ 106.1(4); C(11)-P(1)-Pd(1) 109.0(4); $\mathrm{P}(1)-\mathrm{O}(1)-\mathrm{H}(1 \mathrm{~A})$ 109.5; $\mathrm{Cl}(1)-\mathrm{Pd}(2)-\mathrm{Cl}(2)$ 83.02(9); $\mathrm{Pd}(2)-\mathrm{Cl}(2)-\mathrm{Pd}(1)$ 96.61(9). 


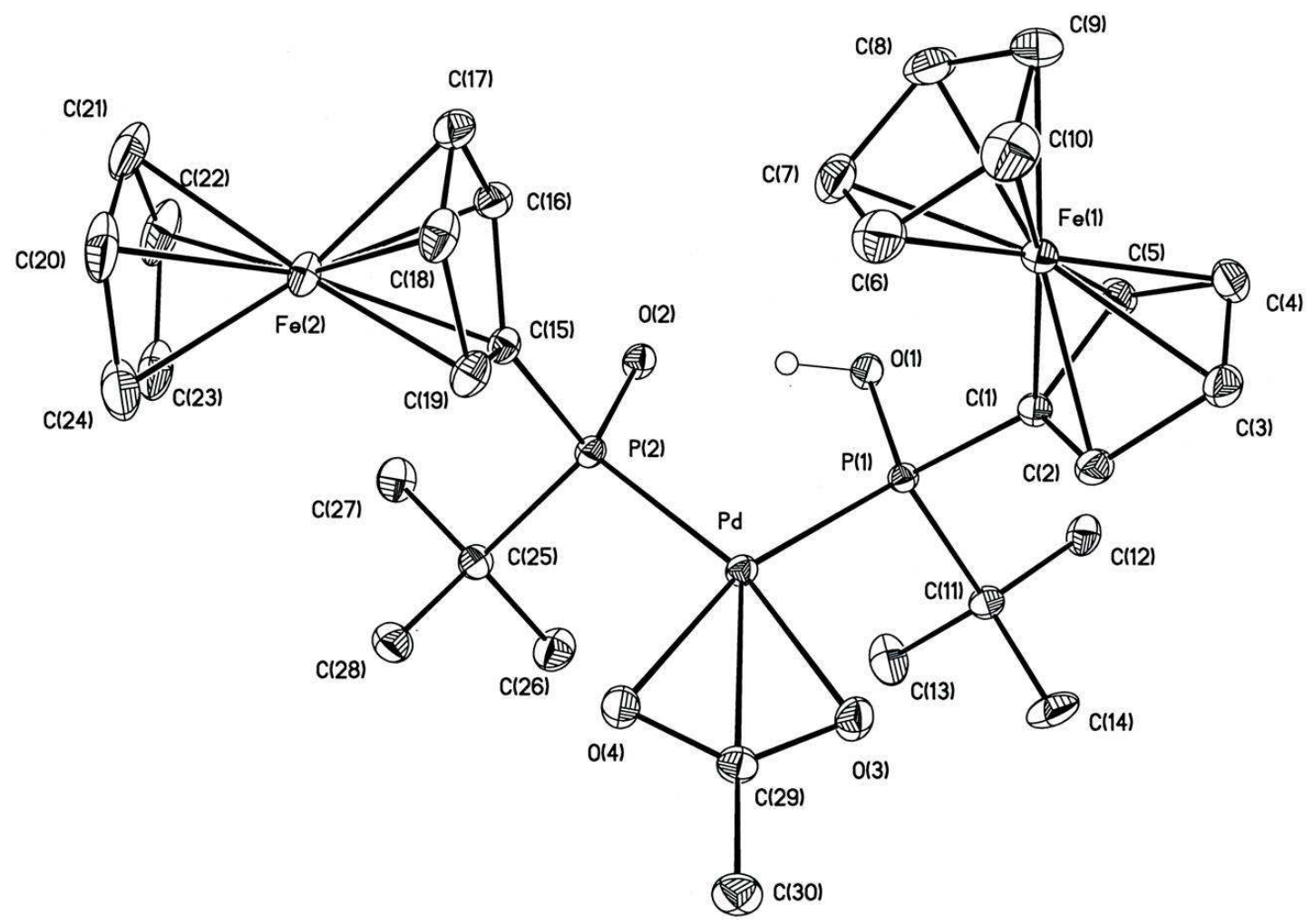

Fig. 5 ORTEP drawing of 9. Hydrogen atoms are omitted for clarity. Selected bond lengths $(\AA)$ and angles $\left({ }^{\circ}\right): \mathrm{Pd}-\mathrm{O}(3)$ 2.181(3); Pd-O(4) 2.194(3); Pd-P(1) 2.2265(11); Pd-P(2) 2.2450(11); Pd-C(29) 2.525(4); $\mathrm{P}(1)-\mathrm{O}(1)$ 1.546(3); $\mathrm{P}(1)-\mathrm{C}(1)$ 1.785(4); $\mathrm{P}(1)-\mathrm{C}(11)$ 1.857(4); $\mathrm{P}(2)-\mathrm{O}(2)$ 1.541(3); $\mathrm{P}(2)-\mathrm{C}(15)$ 1.806(4); $\mathrm{P}(2)-\mathrm{C}(25)$ 1.857(4); $\mathrm{O}(3)-\mathrm{C}(29)$ 1.250(5); O(4)-C(29) 1.263(5); C(29)-C(30) 1.499(6); $\mathrm{O}(3)-\mathrm{Pd}-\mathrm{O}(4)$ 59.67(12); O(3)-Pd-P(1) 103.24(9); O(4)-Pd-P(1) 162.91(9); $\mathrm{O}(3)-\mathrm{Pd}-\mathrm{P}(2)$ 165.23(9); O(4)-Pd-P(2) 105.58(9); P(1)-Pd-P(2) 91.51(4); $\mathrm{O}(3)-\mathrm{Pd}-\mathrm{C}(29)$ 29.66(13); O(4)-Pd-C(29) 30.02(13); P(1)-O(1)-H(1A) 109.5; C(29)-O(4)-Pd 89.6(3); O(3)-C(29)-O(4) 120.1(4); C(30)-C(29)-Pd 178.5(3); $\mathrm{O}(3)-\mathrm{Pd}-\mathrm{O}(4) 59.67(12)$. 
Table 1. Crystal data of $7 \mathbf{a B}, 7 \mathbf{a M}, 7 \mathbf{b M}, 8$ and 9

\begin{tabular}{|c|c|c|c|}
\hline Compound & $7 \mathbf{a B}$ & $7 \mathbf{a M}$ & $7 \mathrm{bM}$ \\
\hline Formula & $\mathrm{C}_{22} \mathrm{H}_{18} \mathrm{FeO}_{2} \mathrm{P}_{2} \bullet \mathrm{H}_{2} \mathrm{O}$ & $\mathrm{C}_{16} \mathrm{H}_{15} \mathrm{FeOP}$ & $\mathrm{C}_{14} \mathrm{H}_{19} \mathrm{FeOP}$ \\
\hline Formula weight & 450.17 & 310.10 & 290.11 \\
\hline Cryst syst & Orthorhombic & Monoclinic & Orthorhombic \\
\hline Space group & Pca2(1) & $\mathrm{P} 21 / \mathrm{c}$ & P212121 \\
\hline$a(\AA)$ & $15.6895(12)$ & $5.8491(12)$ & $5.9833(12)$ \\
\hline$b(\AA)$ & $5.9404(5)$ & $13.243(3)$ & $10.122(2)$ \\
\hline$c(\AA)$ & $22.0284(16)$ & $18.219(4)$ & $22.510(5)$ \\
\hline$\alpha\left(^{\circ}\right)$ & - & - & - \\
\hline$\beta\left(^{\circ}\right)$ & - & $90.840(4)$ & - \\
\hline$\left.x^{\circ}\right)$ & - & - & - \\
\hline$V\left(\AA^{3}\right)$ & 2053.1(3) & $1411.1(5)$ & $1363.2(5)$ \\
\hline$Z$ & 4 & 4 & 4 \\
\hline$D_{c}\left(\mathrm{Mg} / \mathrm{m}^{3}\right)$ & 1.456 & 1.460 & 1.414 \\
\hline$\lambda\left(\mathrm{MoK}_{\alpha}\right), \AA$ & 0.71073 & 0.71073 & 0.71073 \\
\hline$\mu\left(\mathrm{mm}^{-1}\right)$ & 0.910 & 1.170 & 1.205 \\
\hline$\theta$ range $\left(^{\circ}\right)$ & 2.60 to $26.04^{\circ}$ & 1.90 to 26.04 & 1.81 to 26.02 \\
\hline Observed reflections $(\mathrm{F}>4 \sigma(\mathrm{F}))$ & 3364 & 2773 & 2680 \\
\hline No. of refined parameters & 261 & 188 & 158 \\
\hline${ }^{\mathrm{a}} R_{1}$ for significant reflctns & 0.0436 & 0.0327 & 0.0298 \\
\hline${ }^{\mathrm{b}} w R_{2}$ for sigft reflctns & 0.1100 & 0.0947 & 0.0843 \\
\hline${ }^{\mathrm{c}} \mathrm{GoF}$ & 1.104 & 0.929 & 0.871 \\
\hline
\end{tabular}


(Continued)

Table 1. Crystal data of $7 \mathbf{a B}, 7 \mathbf{a M}, 7 \mathbf{b M}, 8$ and 9

\begin{tabular}{lll} 
Compound & $\mathbf{8}$ & $\mathbf{9}$ \\
Formula & $\mathrm{C}_{60} \mathrm{H}_{84} \mathrm{Cl}_{2} \mathrm{Fe}_{4} \mathrm{O}_{5} \mathrm{P}_{4} \mathrm{Pd}_{2}$ & $\mathrm{C}_{30} \mathrm{H}_{0} \mathrm{Fe}_{2} \mathrm{O}_{4} \mathrm{P}_{2} \mathrm{Pd}$ \\
Formula weight & 1516.25 & 744.66 \\
Cryst syst & Monoclinic & Triclinic \\
Space group & $\mathrm{Cc}$ & $\mathrm{P}-1$ \\
$a(\AA)$ & $23.058(3)$ & $9.1244(12)$ \\
$b(\AA)$ & $20.034(2)$ & $10.3835(13)$ \\
$c(\AA)$ & $16.7798(18)$ & $16.218(2)$ \\
$\alpha\left(^{\circ}\right)$ & - & $96.204(2)$ \\
$\beta\left({ }^{\circ}\right)$ & $124.311(2)$ & $94.089(2)$ \\
$\left.\chi^{\circ}\right)$ & - & $91.695(2)$ \\
$V\left(\AA^{3}\right)$ & $6402.6(12)$ & $1522.6(3)$ \\
$Z$ & 4 & 2 \\
$D_{c}\left(\mathrm{Mg} / \mathrm{m}^{3}\right)$ & 1.573 & 1.624 \\
$\lambda(\mathrm{MoK} \alpha), \AA$ & 0.71073 & 0.71073 \\
$\mu\left(\mathrm{mm}{ }^{-1}\right)$ & 1.661 & 1.663 \\
$\theta$ range $\left({ }^{\circ}\right)$ & 1.48 to 26.05 & 1.27 to 26.06 \\
Observed reflections $(\mathrm{F}>4 \sigma(\mathrm{F}))$ & 9217 & 5939 \\
No. of refined parameters & 627 & 352 \\
${ }^{\mathrm{a}} R_{1}$ for significant reflctns & 0.0552 & 0.0407 \\
${ }^{\mathrm{b}} w R_{2}$ for sigft reflctns & 0.1378 & 0.1044 \\
${ }^{\mathrm{c}} \mathrm{GoF}$ & 1.035 & 1.016 \\
& & \\
\hline
\end{tabular}

${ }^{\mathrm{a}} R_{1}=\left|\Sigma\left(\left|F_{\mathrm{o}}\right|-\left|F_{\mathrm{c}}\right|\right) /\right| \Sigma F_{\mathrm{o}} \|$.

${ }^{\mathrm{b}} w R_{2}=\left\{\Sigma\left[\mathrm{w}\left(F_{\mathrm{o}}{ }^{2}-F_{\mathrm{c}}{ }^{2}\right)^{2} / \Sigma\left[\mathrm{w}\left(F_{\mathrm{o}}{ }^{2}\right)^{2}\right\}\right\}^{1 / 2} ; \mathrm{w}=0.0700,0.0800,0.0800,0.0700\right.$ and 0.0750 for crystal data of $7 \mathbf{a B}, 7 \mathbf{a M}, 7 \mathbf{b M}, 8$ and 9 , respectively.

${ }^{\mathrm{c}} \mathrm{GoF}=\left[\Sigma \mathrm{w}\left(F_{\mathrm{o}}{ }^{2}-F_{\mathrm{c}}{ }^{2}\right)^{2} /\left(N_{\mathrm{rflns}}-N_{\text {params }}\right)\right]^{1 / 2}$. 


\section{中興大學研發處貴重儀器使用中心}

元素分析儀 Elementar vario EL III CHN-OS Rapid 服務報告書 N0.1

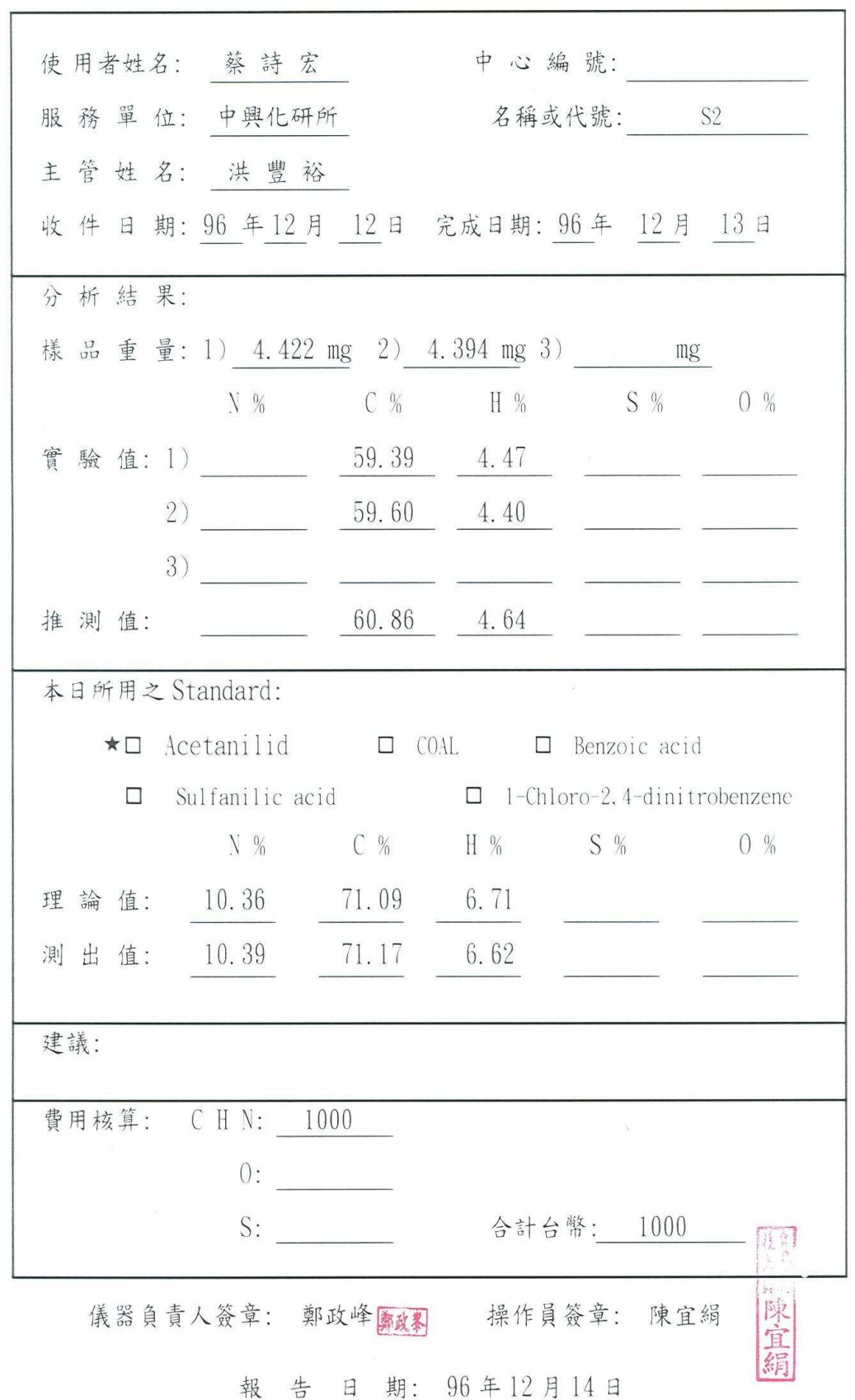

Elemental Analysis of 7aB:

Anal. Calcd.: C, 60.86; H, 4.64.

Found: C, 59.50; H, 4.44. 


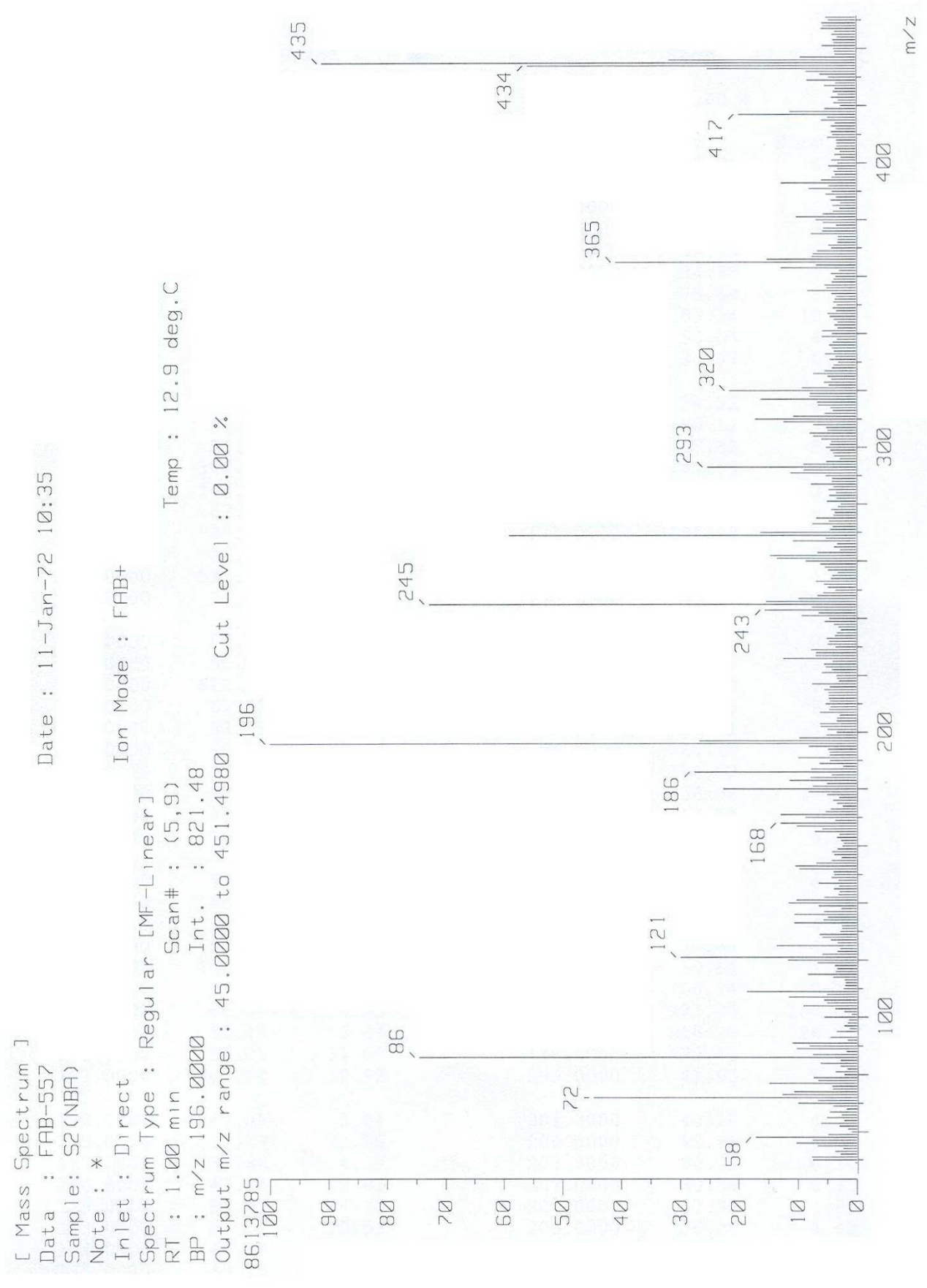

MS of $7 \mathbf{a B}$ 


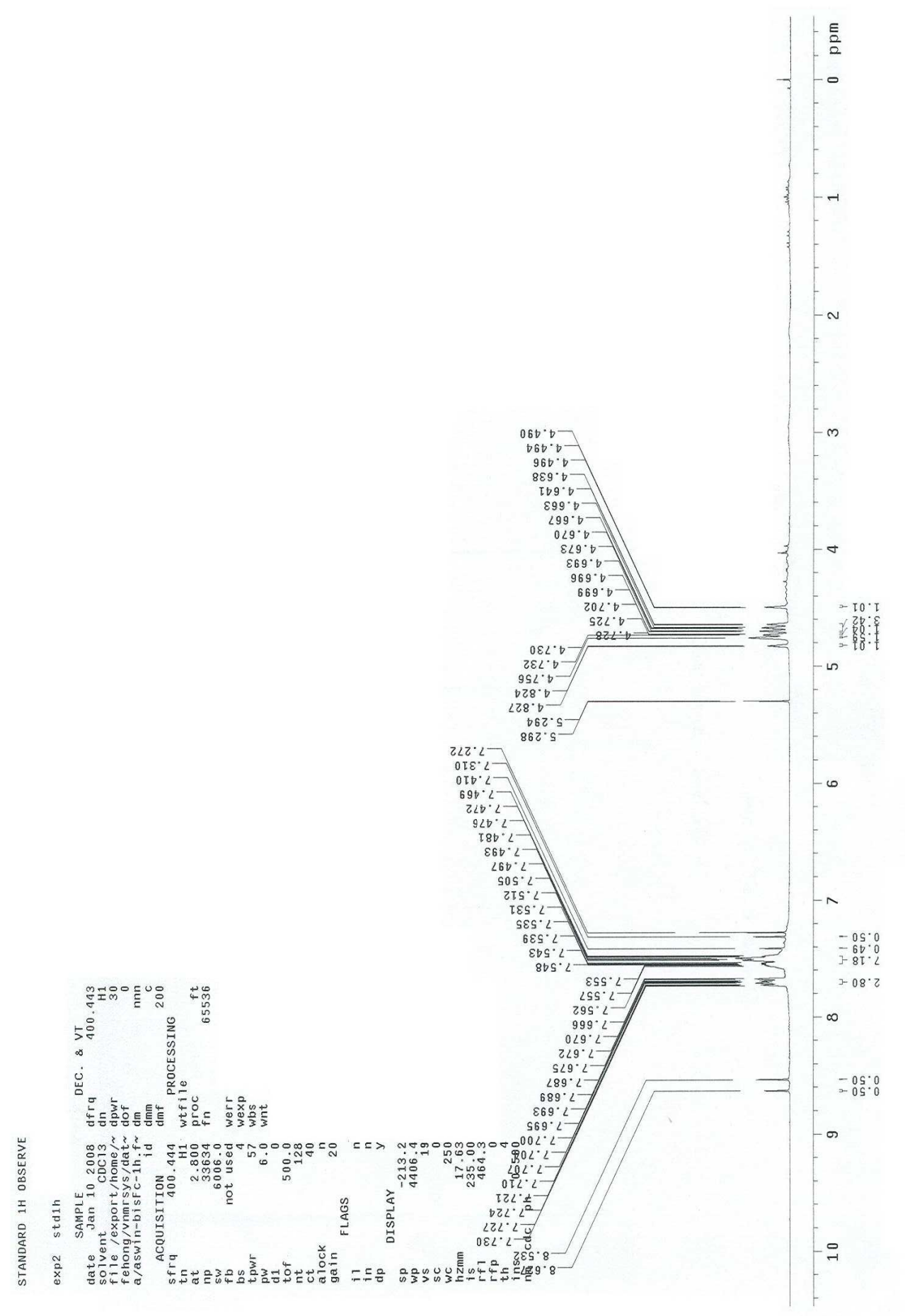

${ }^{1} \mathrm{H}$ NMR of $\mathbf{7 a B}$ 


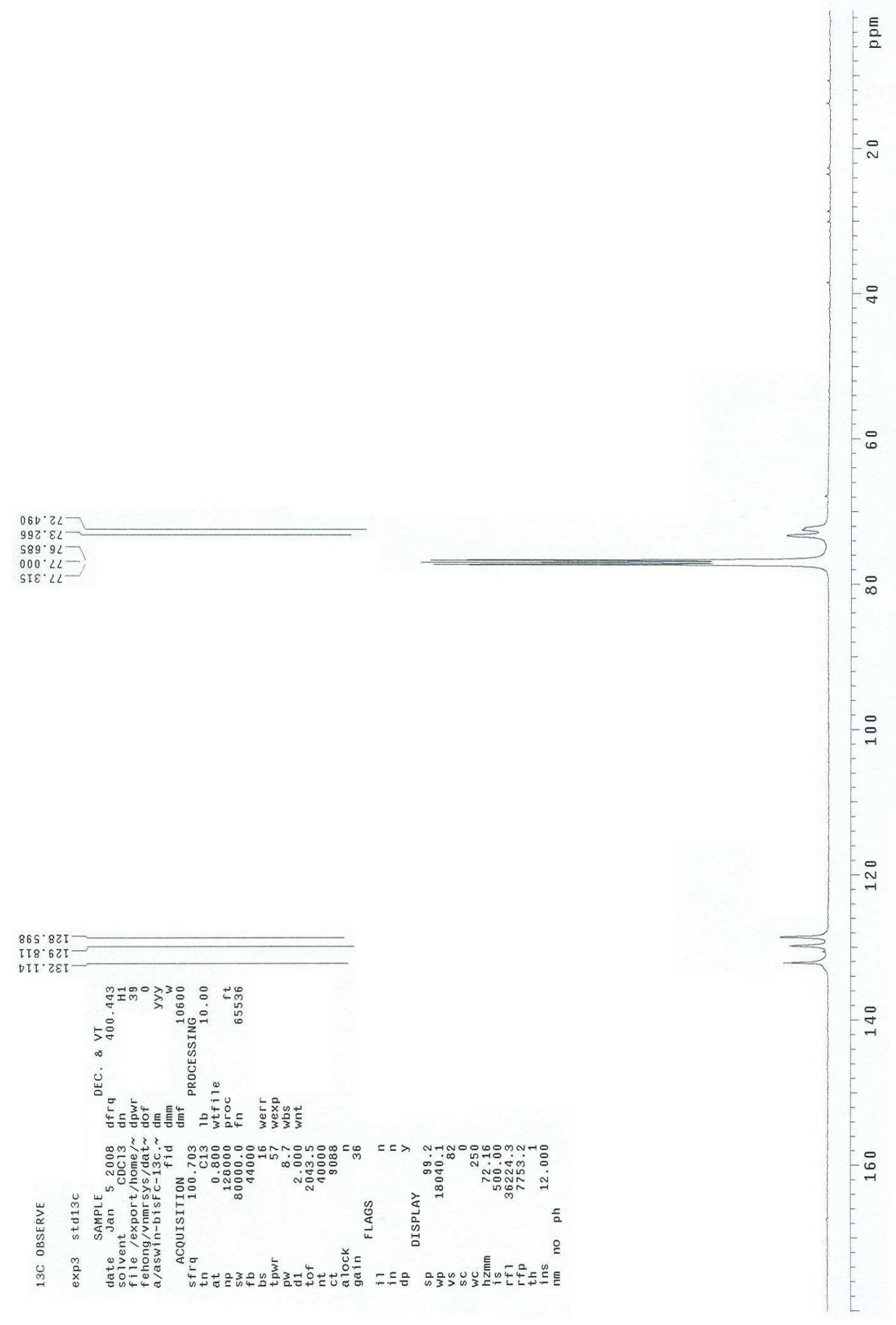

${ }^{13} \mathrm{C}$ NMR of $7 \mathbf{a B}$ 


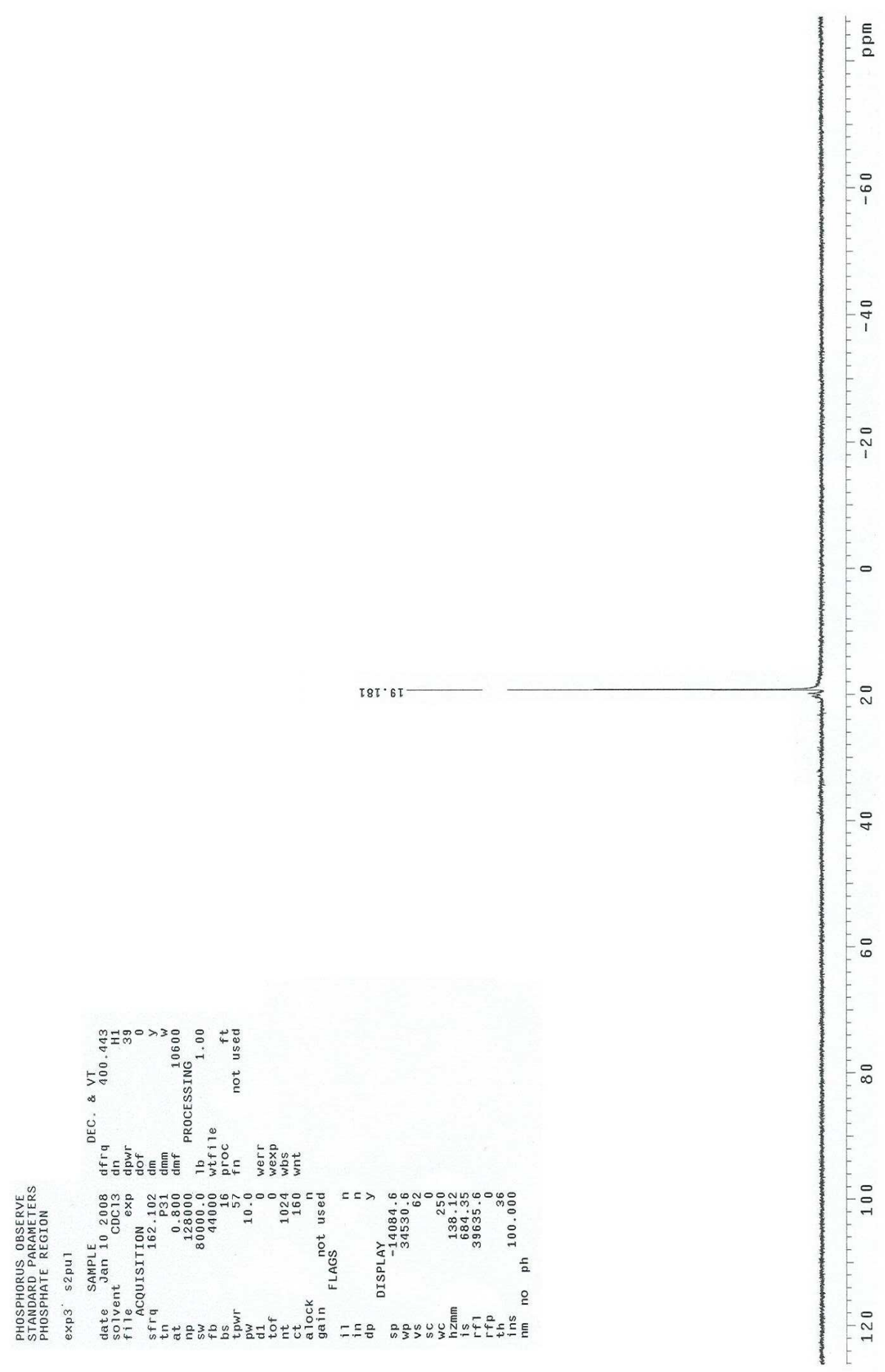

${ }^{31} \mathrm{P}$ NMR of $7 \mathbf{a B}$ 


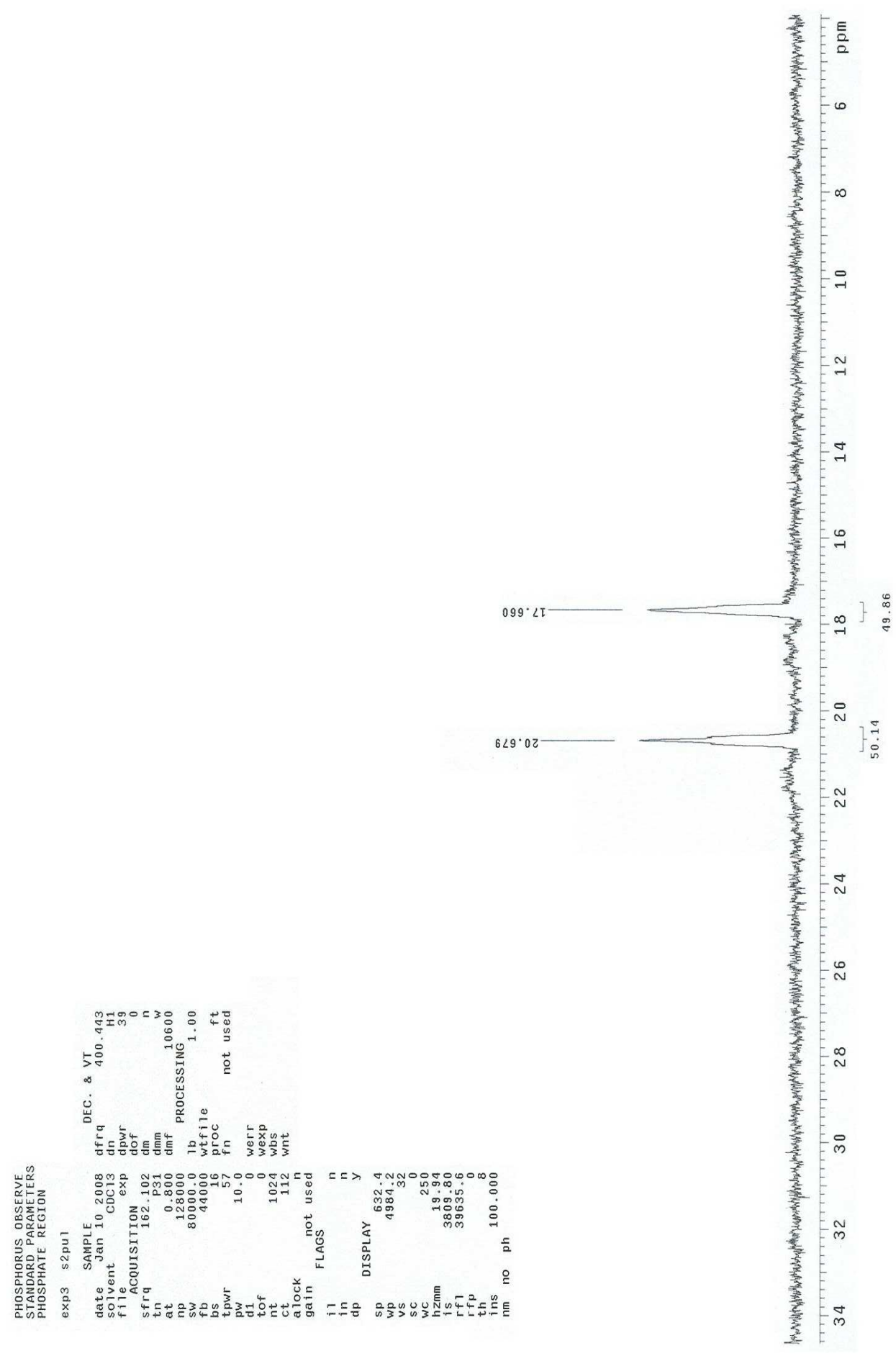

${ }^{31}$ P NMR of $7 \mathbf{a B}$ (coupled) 


\section{中興大學研發處貴重儀器使用中心}

元素分析儀 Elementar vario EL III CHN-OS Rapid 服務報告書 N0.1

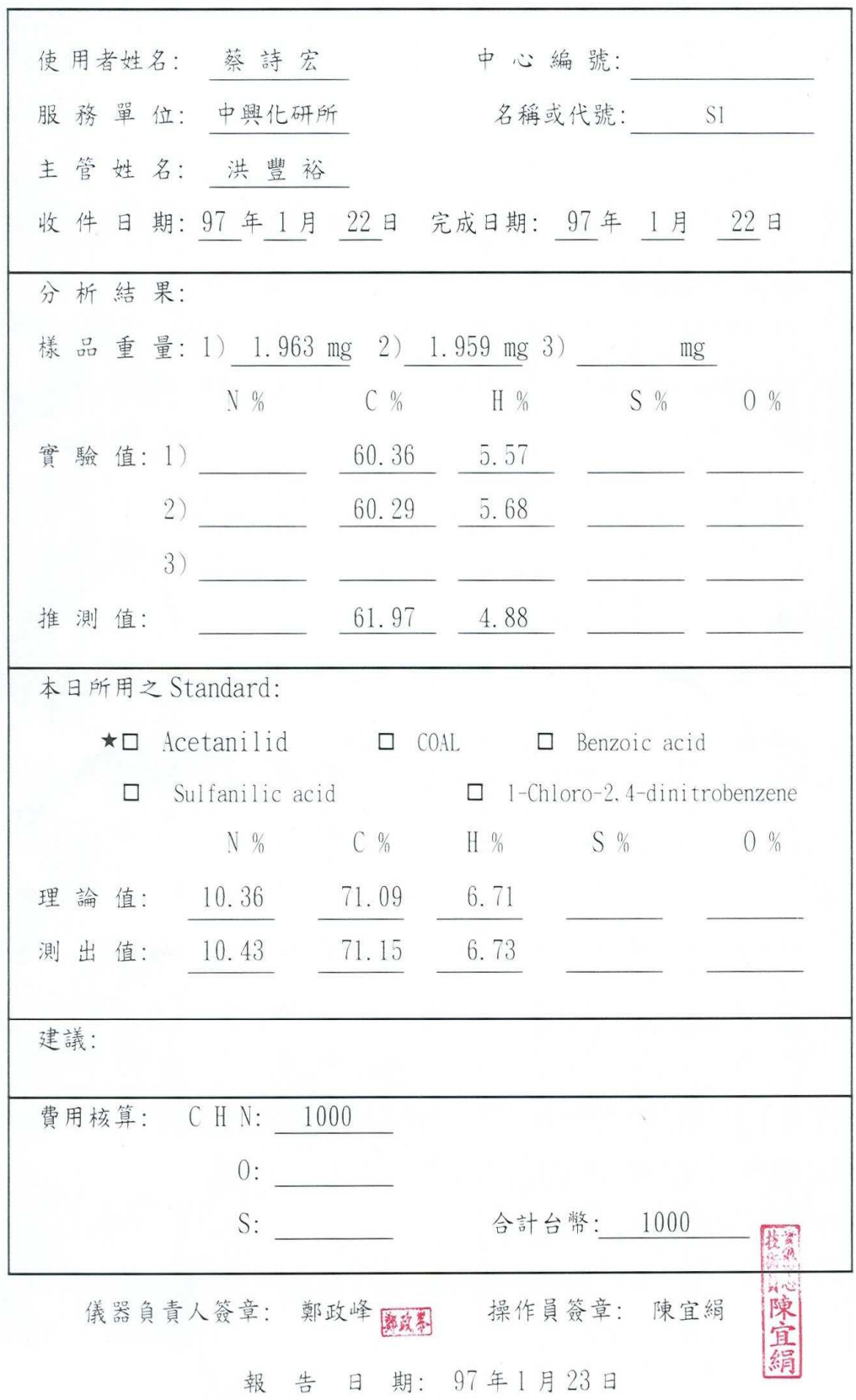

\section{Elemental Analysis of 7aM:}

Anal. Calcd.: C, 61.97; H, 4.88.

Found: C, 60.33; H, 5.63. 


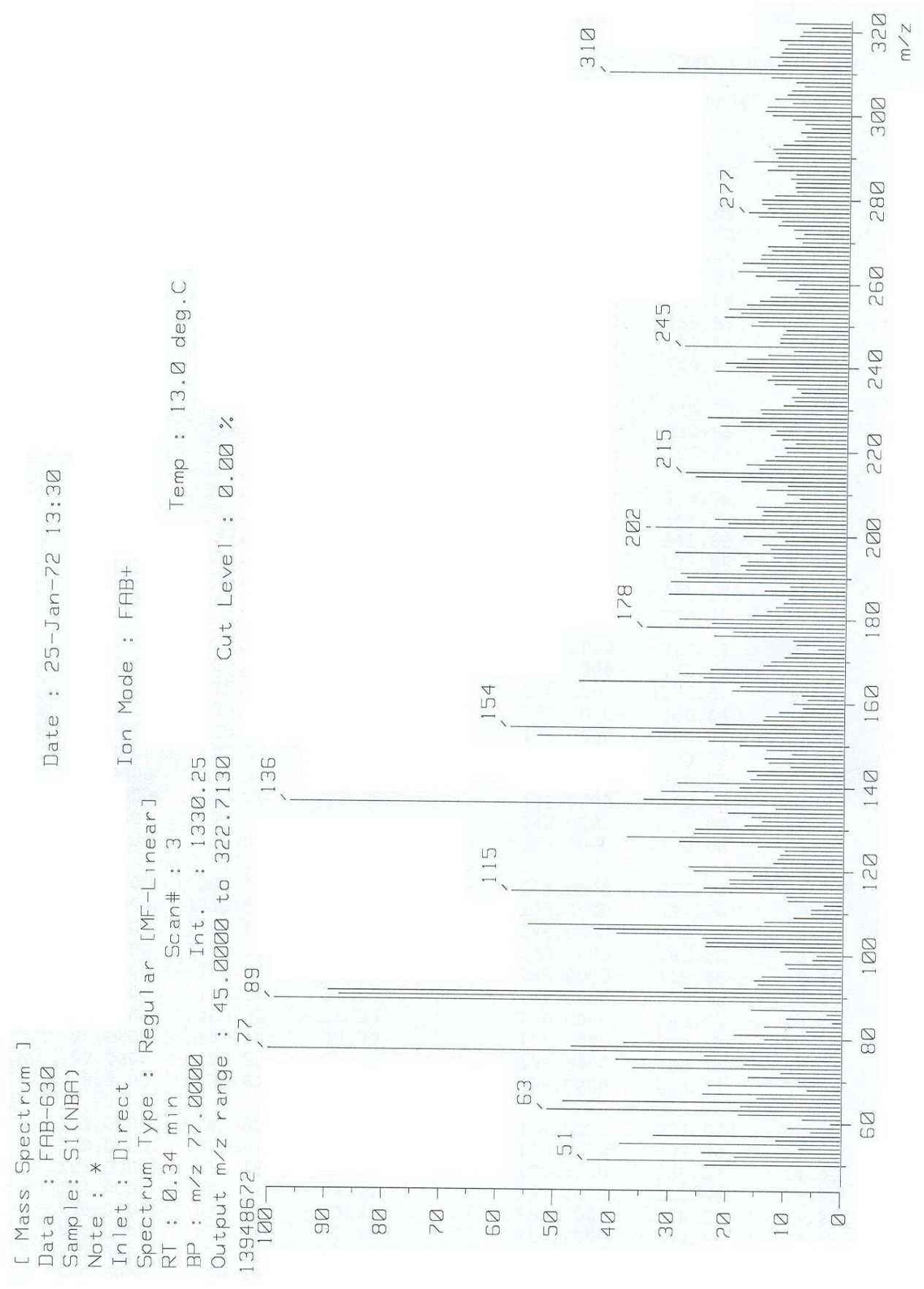

MS of 7aM 


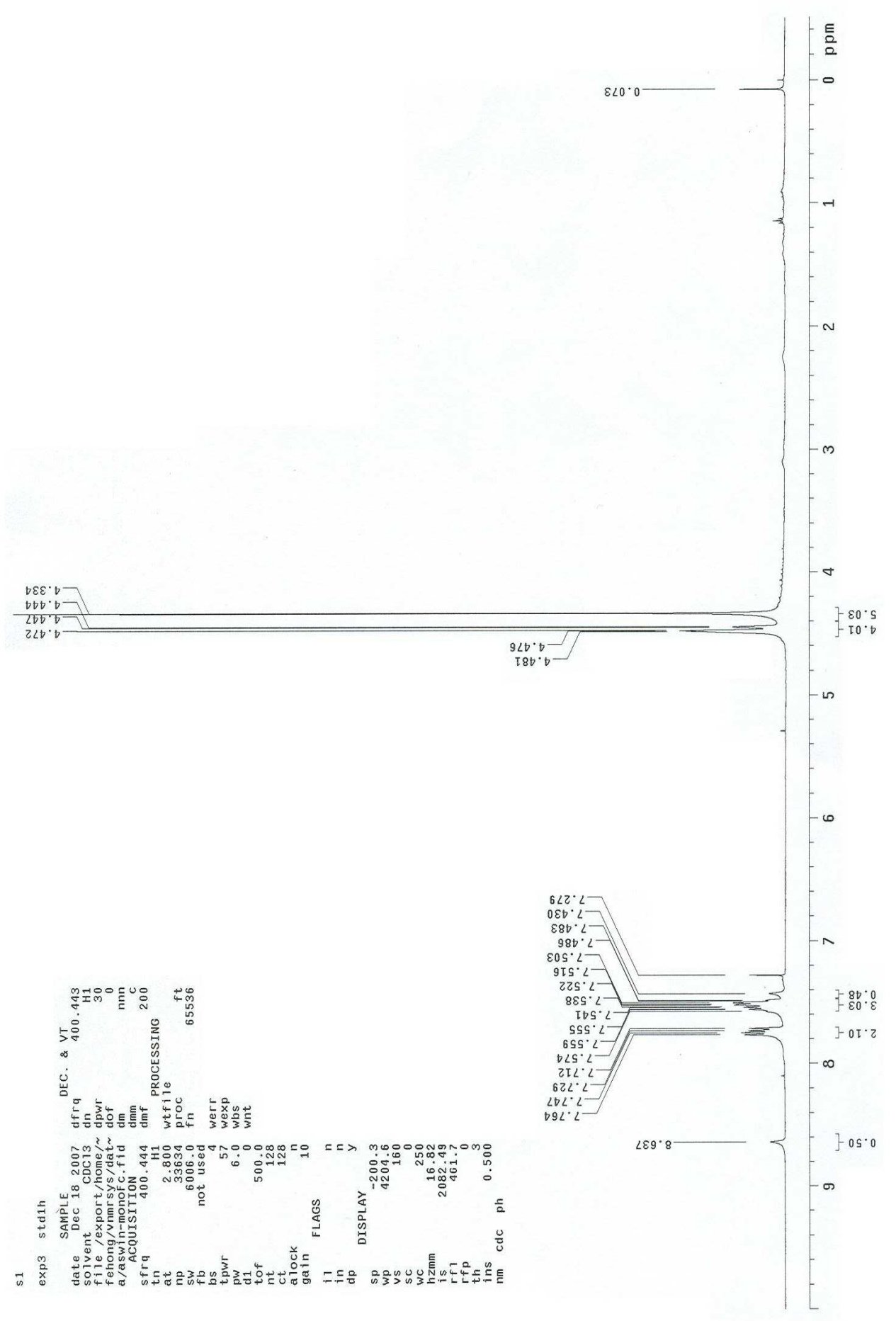

${ }^{1} \mathrm{H}$ NMR of $7 \mathbf{a M}$ 


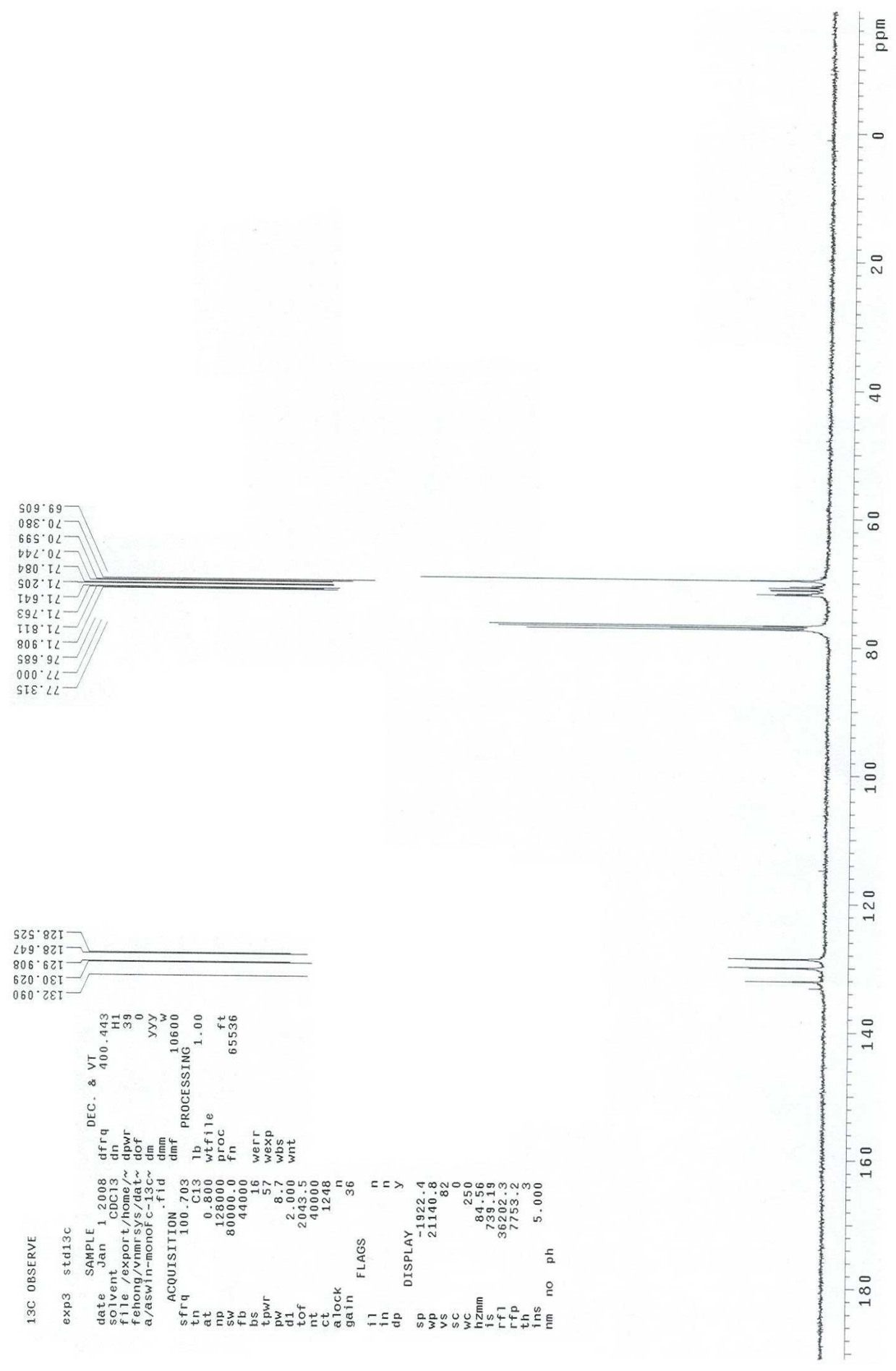

${ }^{13} \mathrm{C}$ NMR of $7 \mathbf{a M}$ 


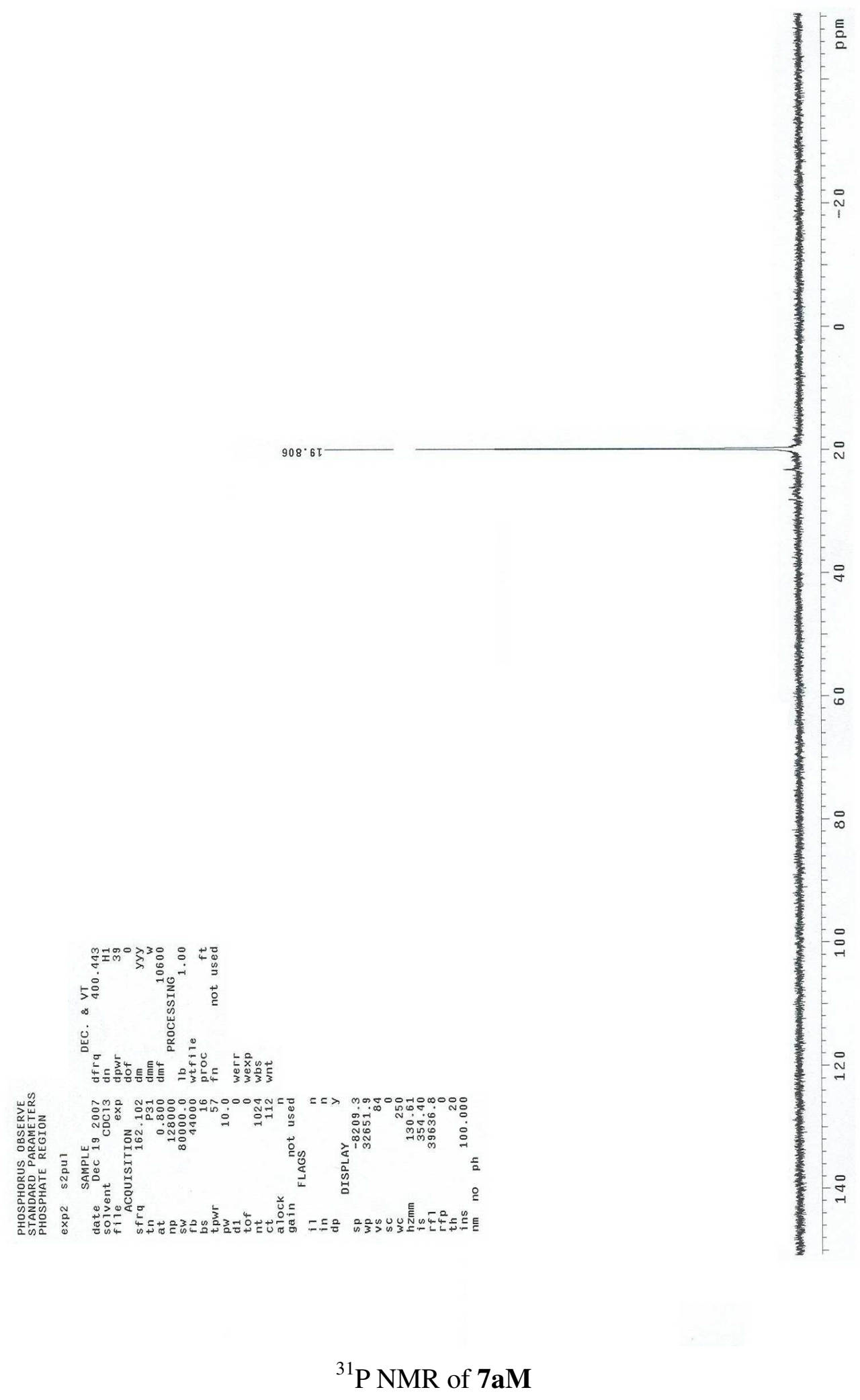




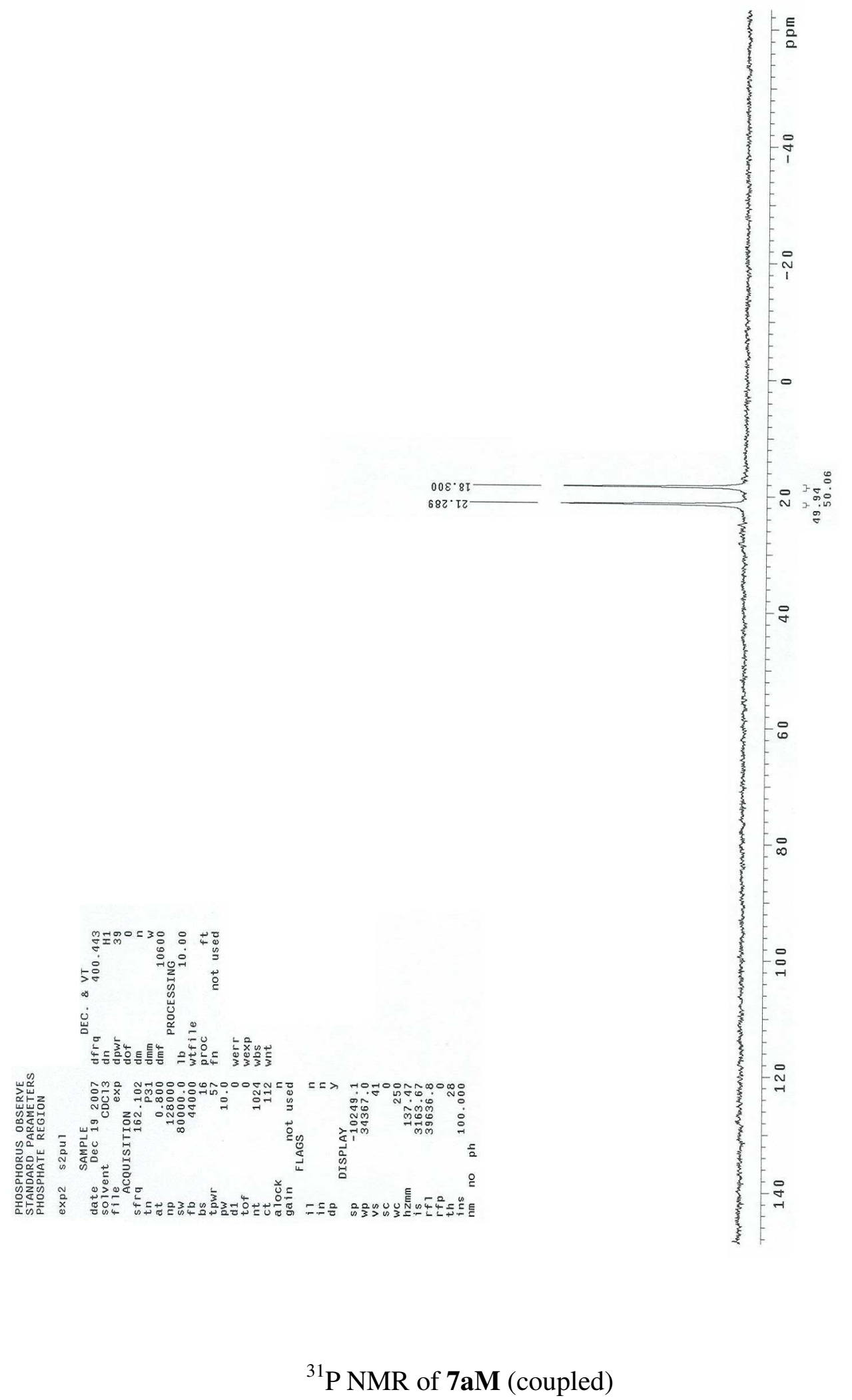




\section{中興大學研發處貴重儀器使用中心}

元素分析儀 Elementar vario EL III CHN-OS Rapid 服務報告書 NO.1

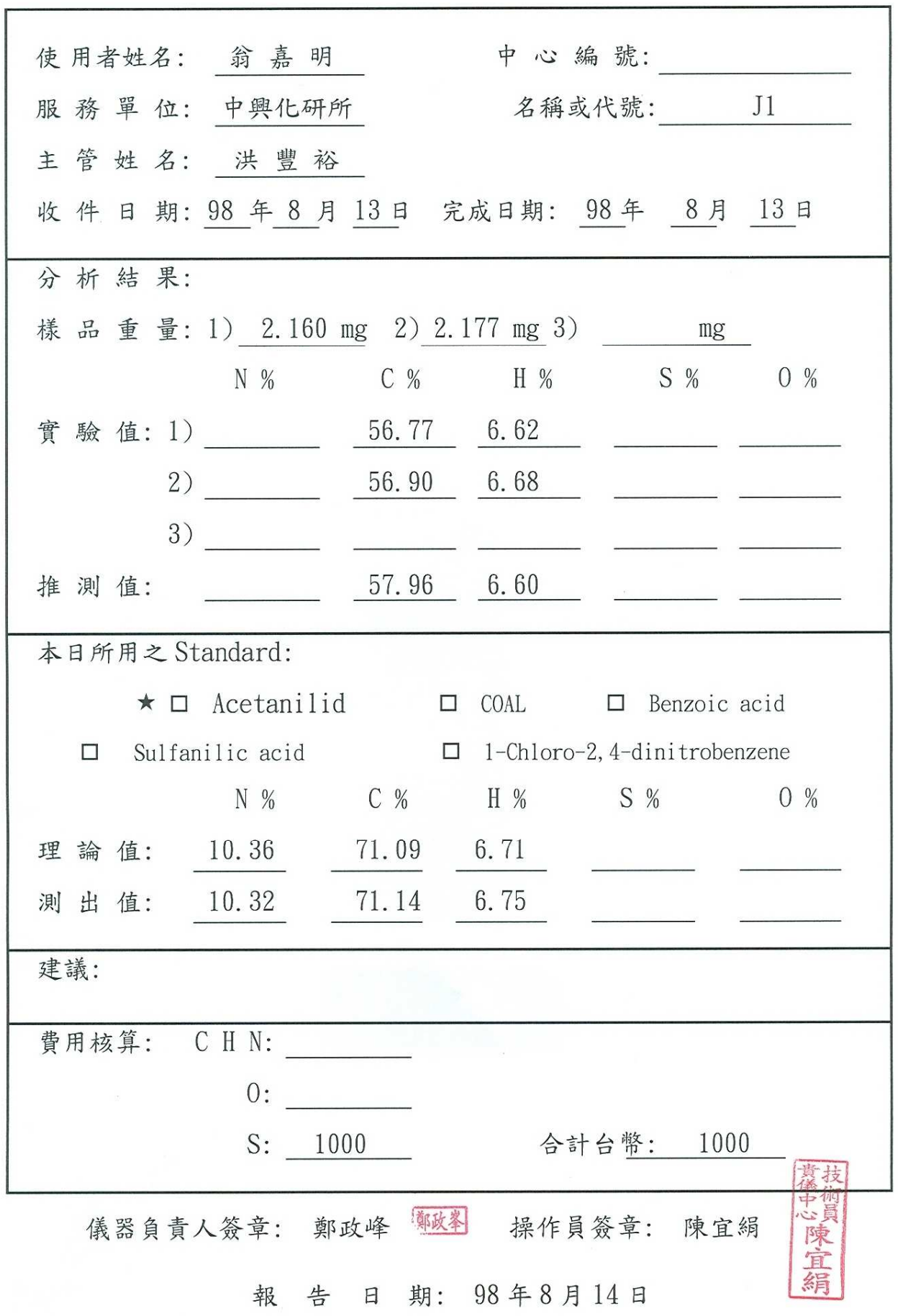

Elemental Analysis of 7bM:

Anal. Calcd.: C, 57.96; H, 6.60.

Found: C, 56.84; H, 6.65 . 


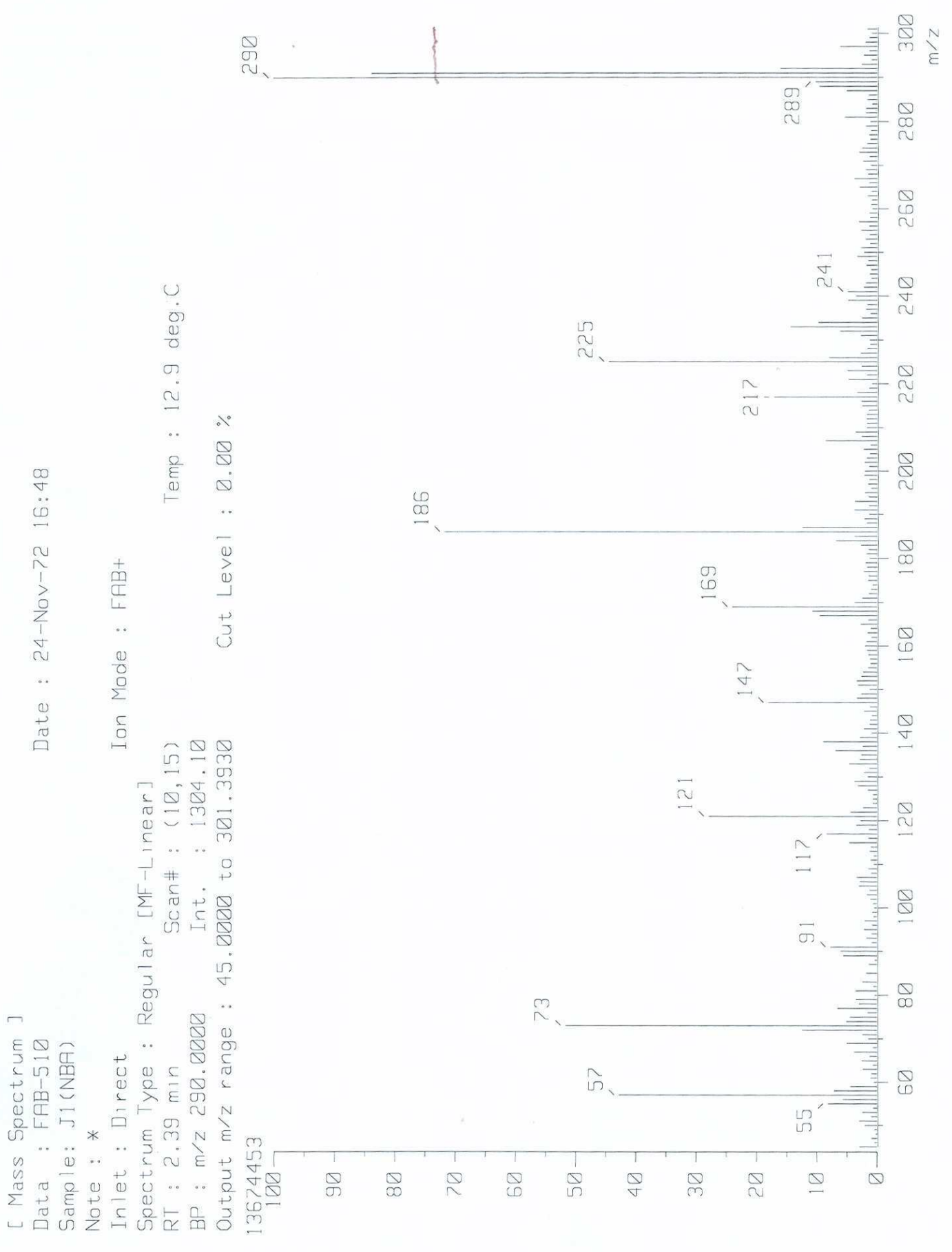

MS of $7 \mathbf{b M}$ 


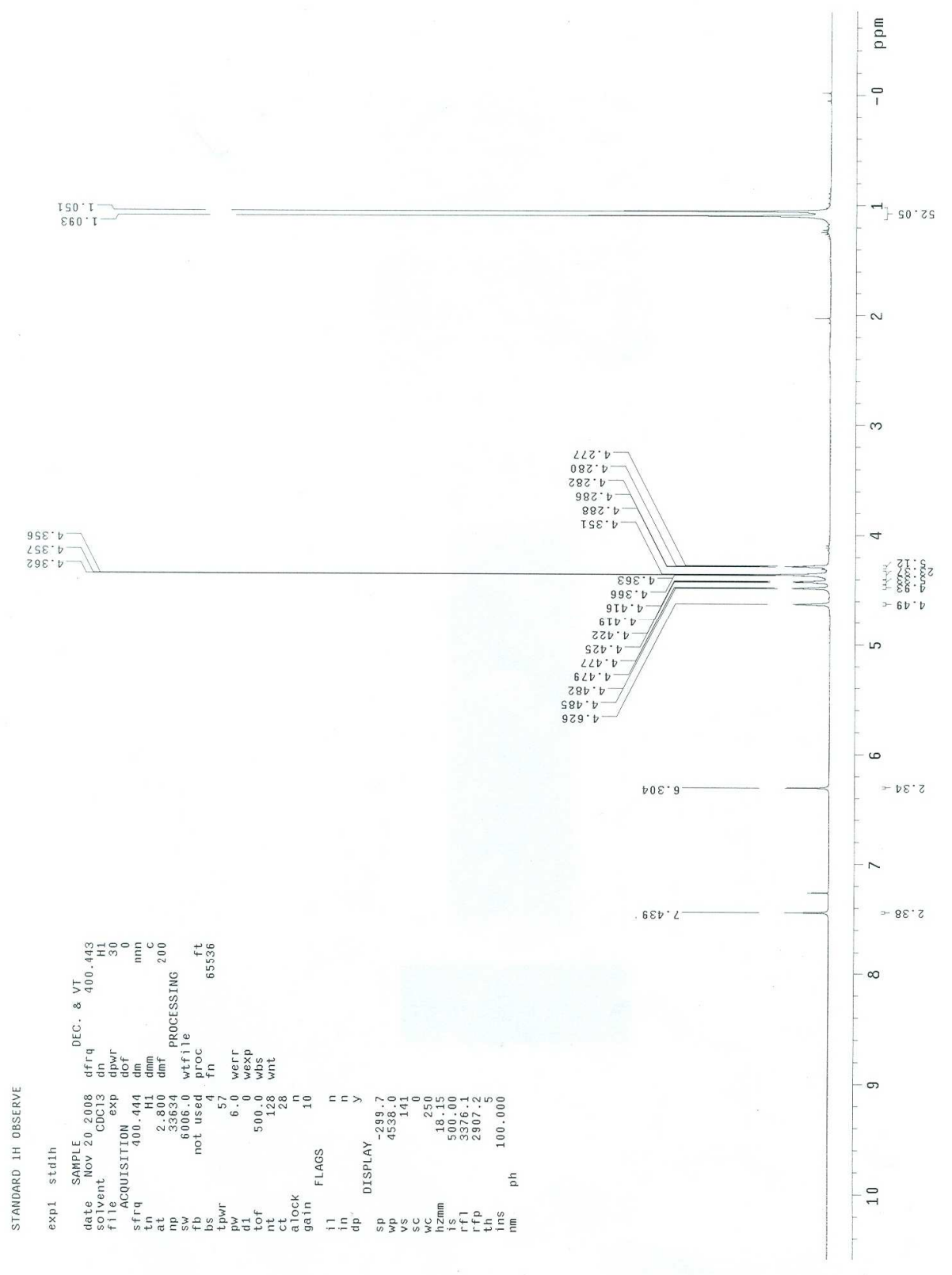

${ }^{1} \mathrm{H}$ NMR of $\mathbf{7 b M}$ 


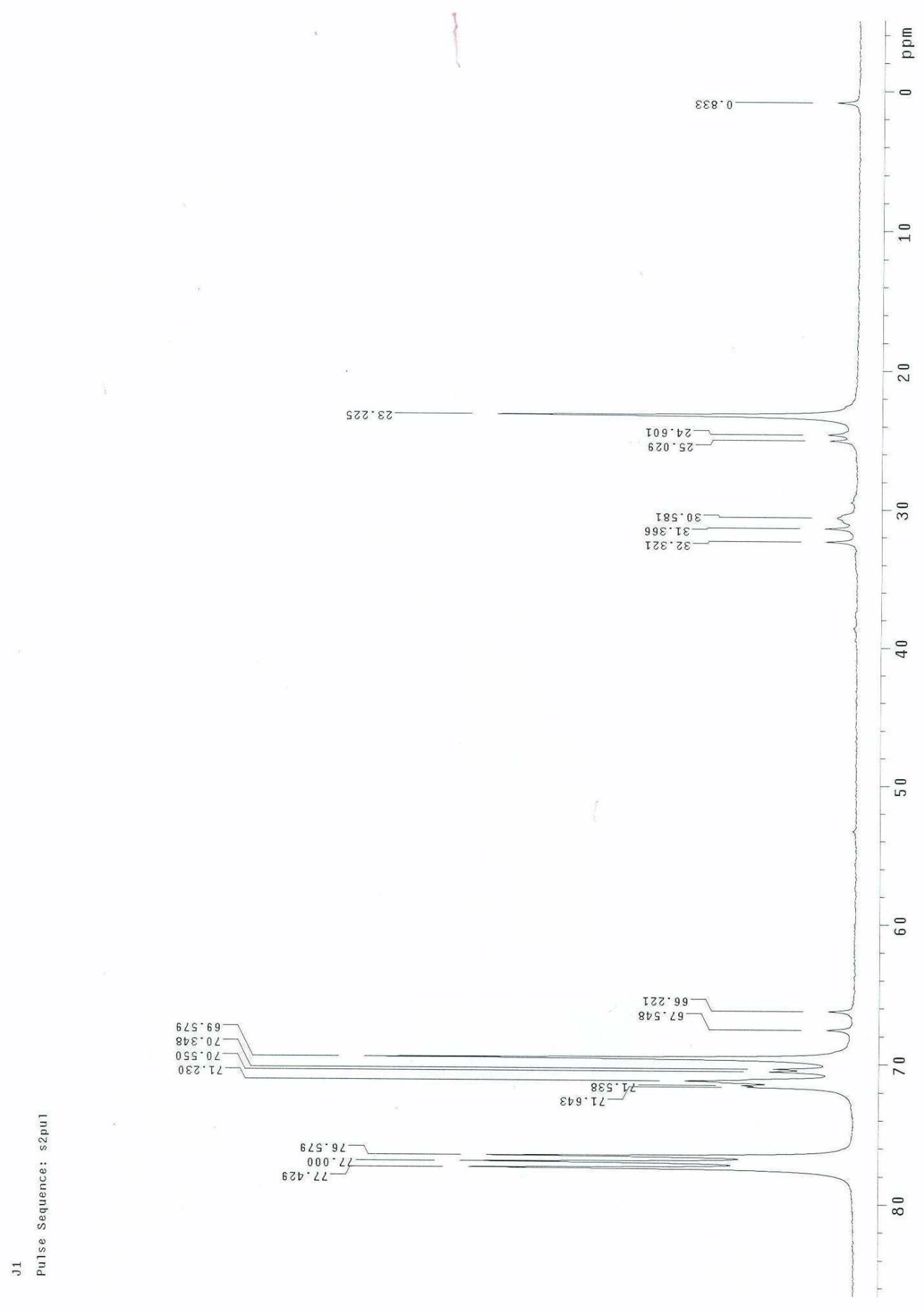

${ }^{13} \mathrm{C}$ NMR of $7 \mathbf{b M}$ 


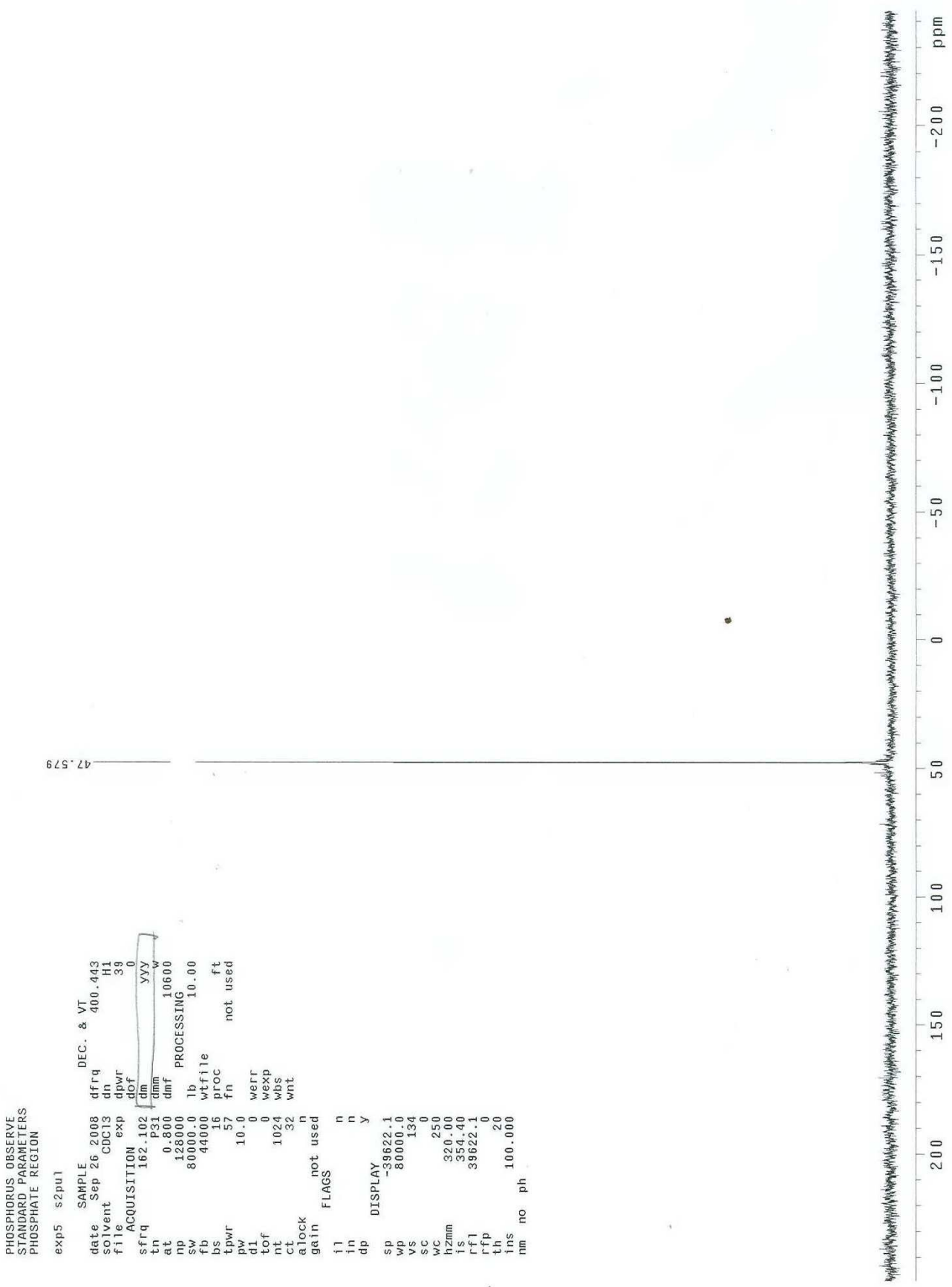

${ }^{31}$ P NMR of $7 \mathbf{b M}$

$-25-$ 


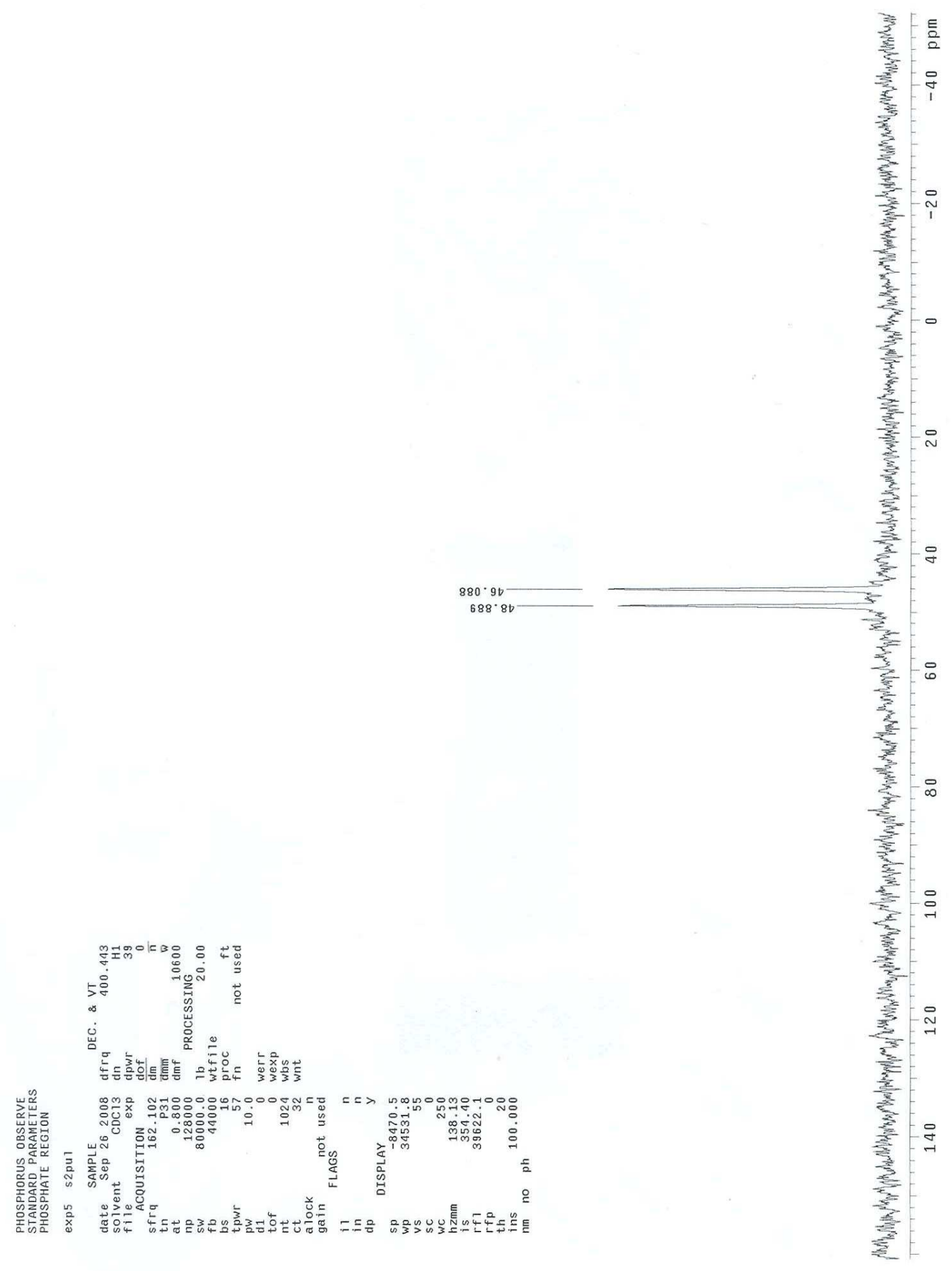

${ }^{31} \mathrm{P}$ NMR of $\mathbf{7 b M}$ (coupled) 


\section{中興大學研發處貴重儀器使用中心}

元素分析儀 Elementar vario EL III CHN-OS Rapid 服務報告書 NO. 1

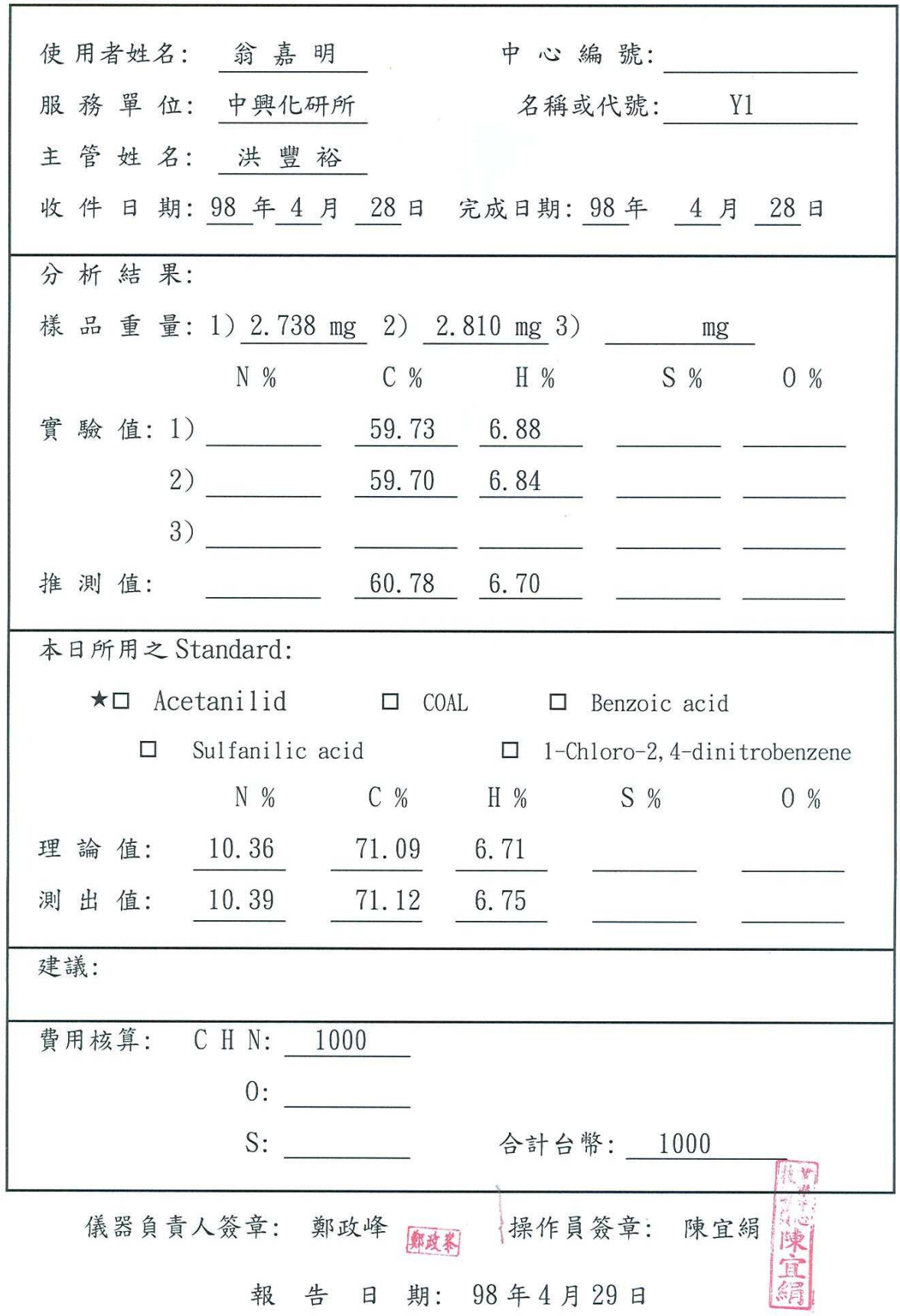

Elemental Analysis of 7cM:

Anal. Calcd.: C, 60.78; H, 6.70.

Found: C,59.72; H,6.86. 


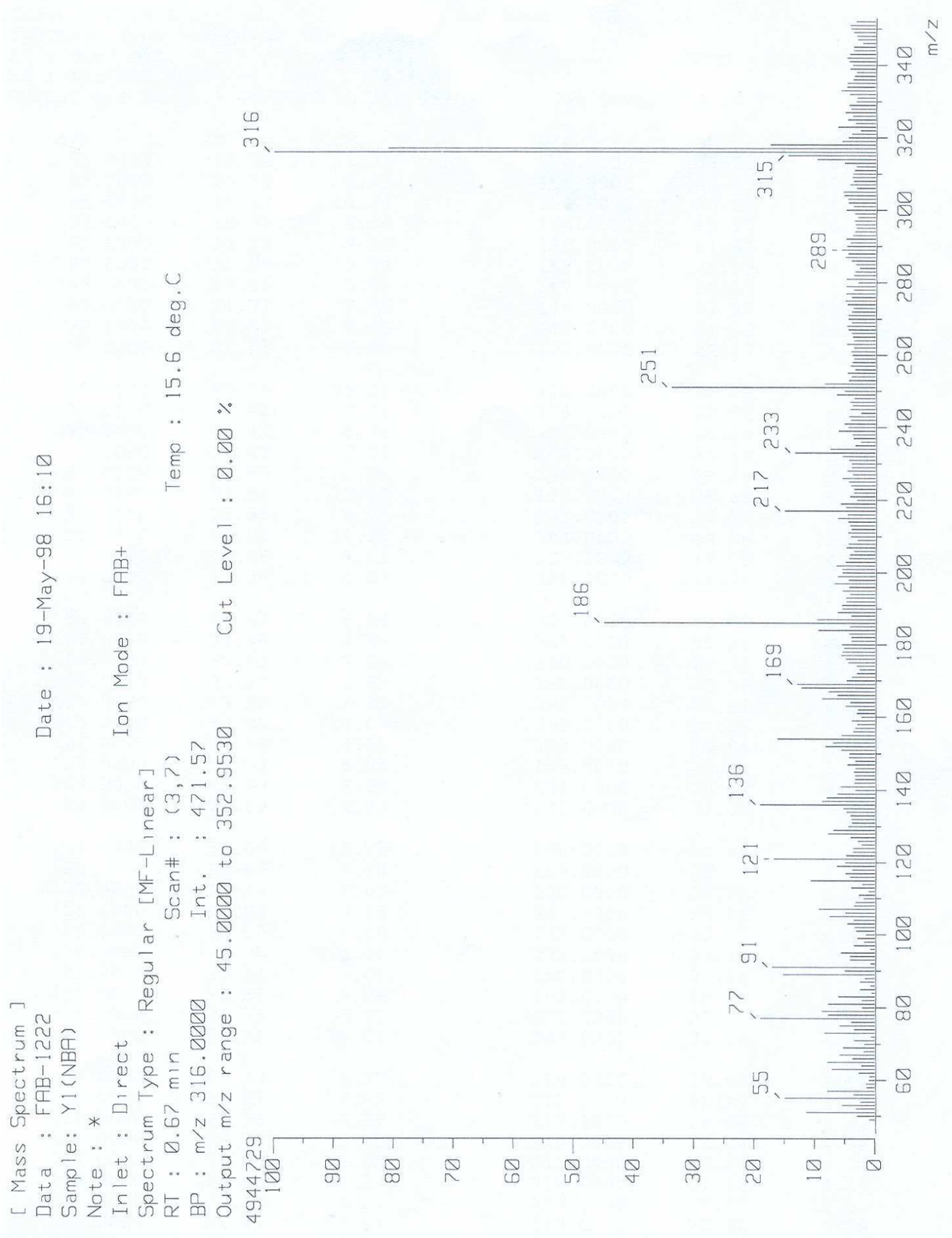

MS of 7cM 


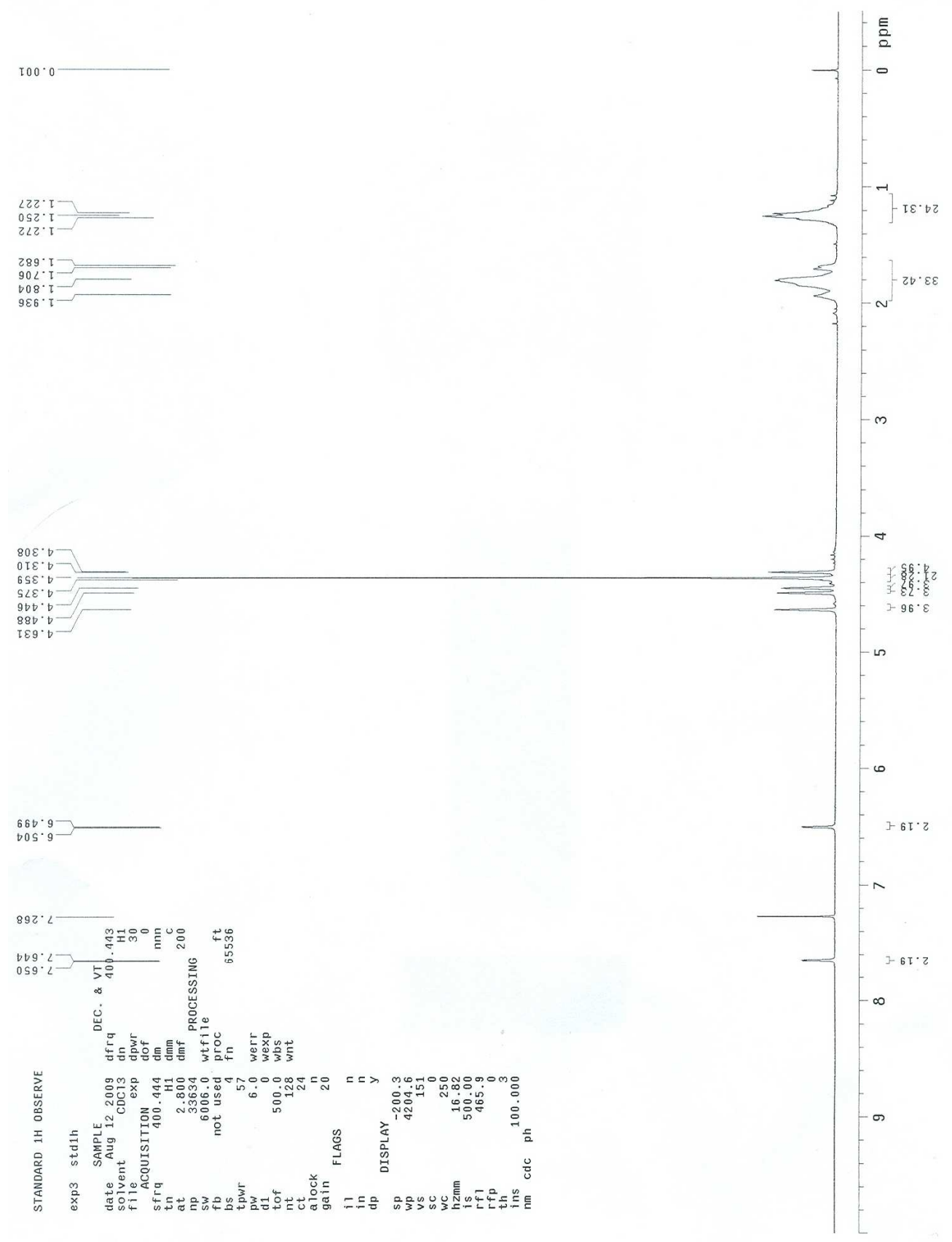

${ }^{1} \mathrm{H}$ NMR of $\mathbf{7 c M}$ 


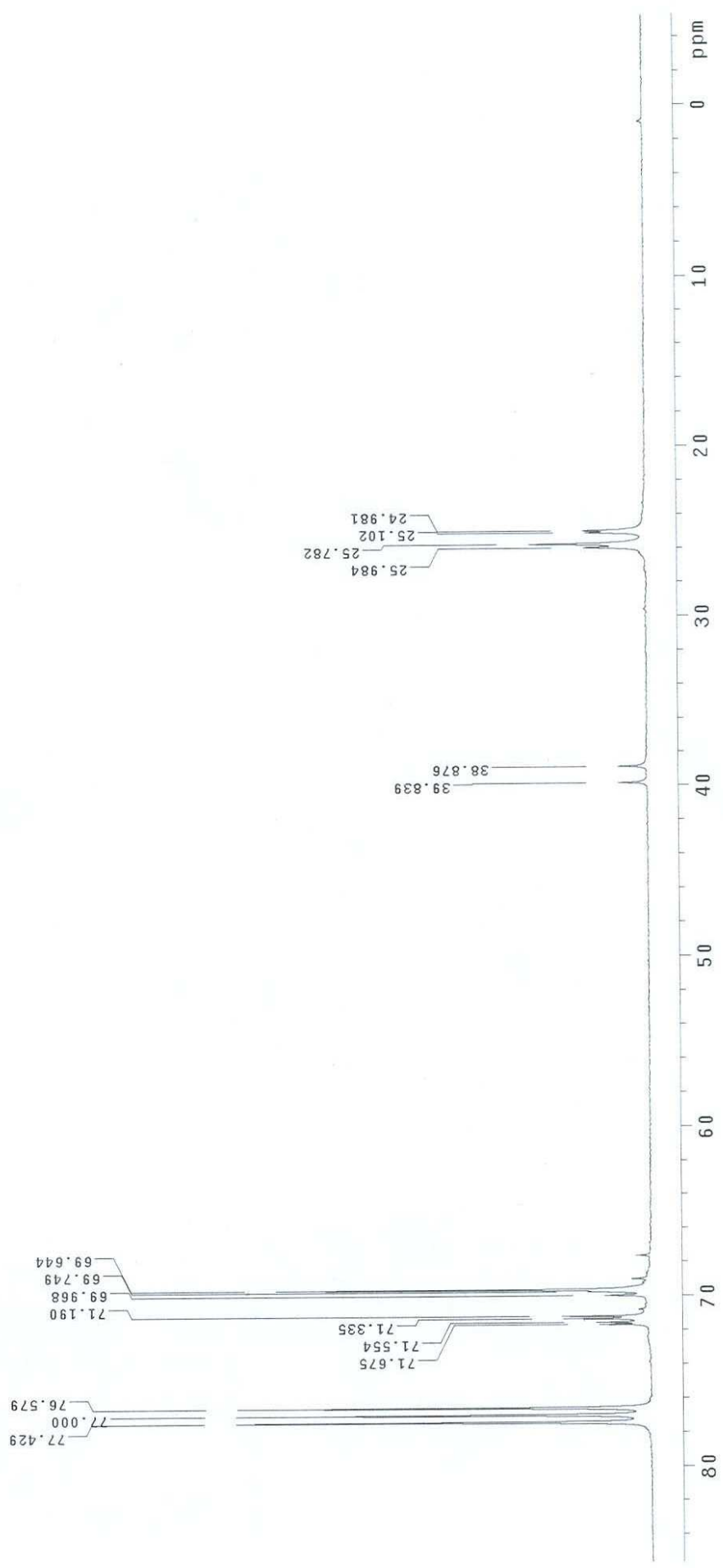

${ }^{13} \mathrm{C}$ NMR of $7 \mathbf{c M}$

$-30-$ 


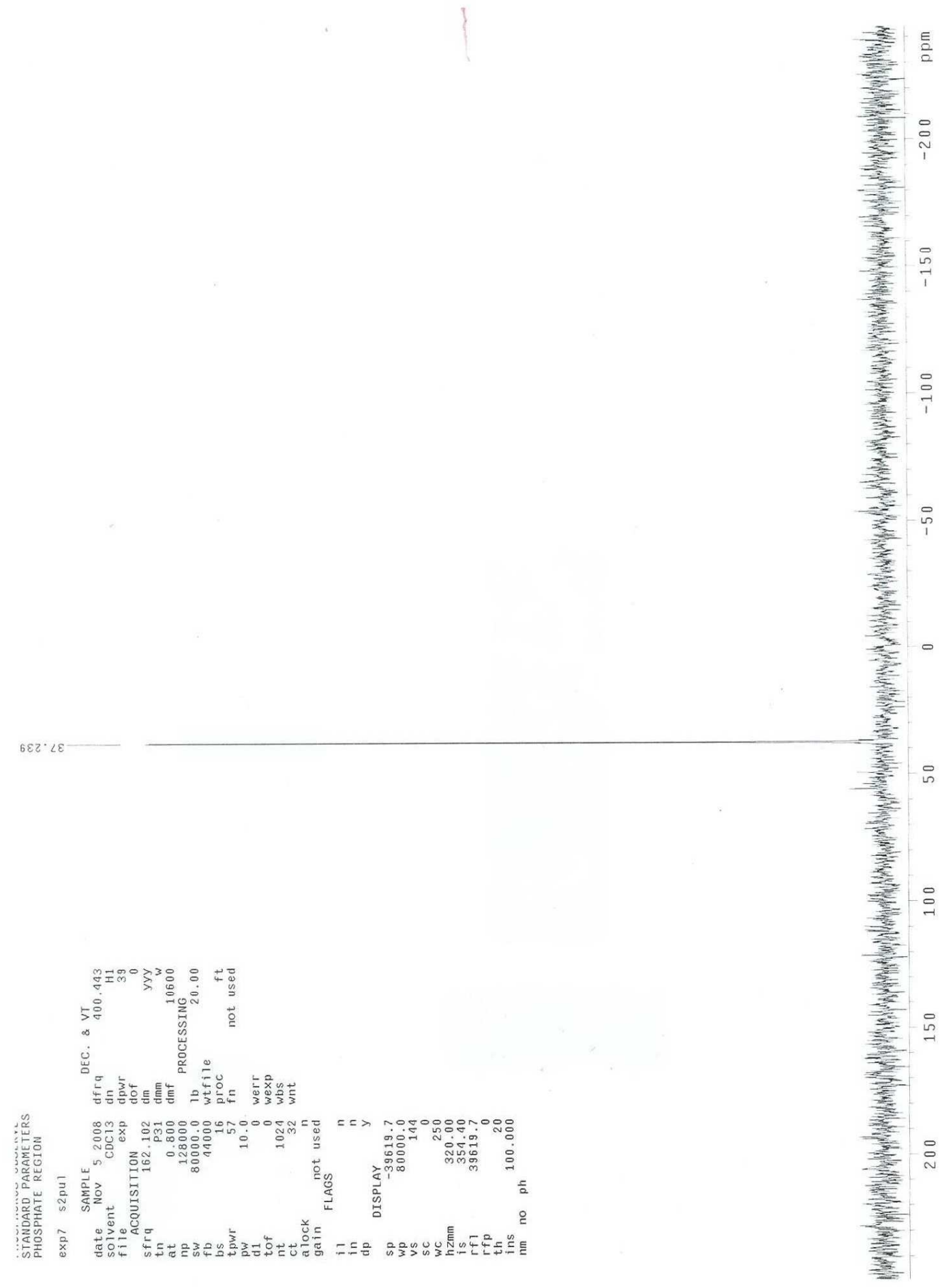

${ }^{31} \mathrm{P}$ NMR of $7 \mathbf{c M}$ 


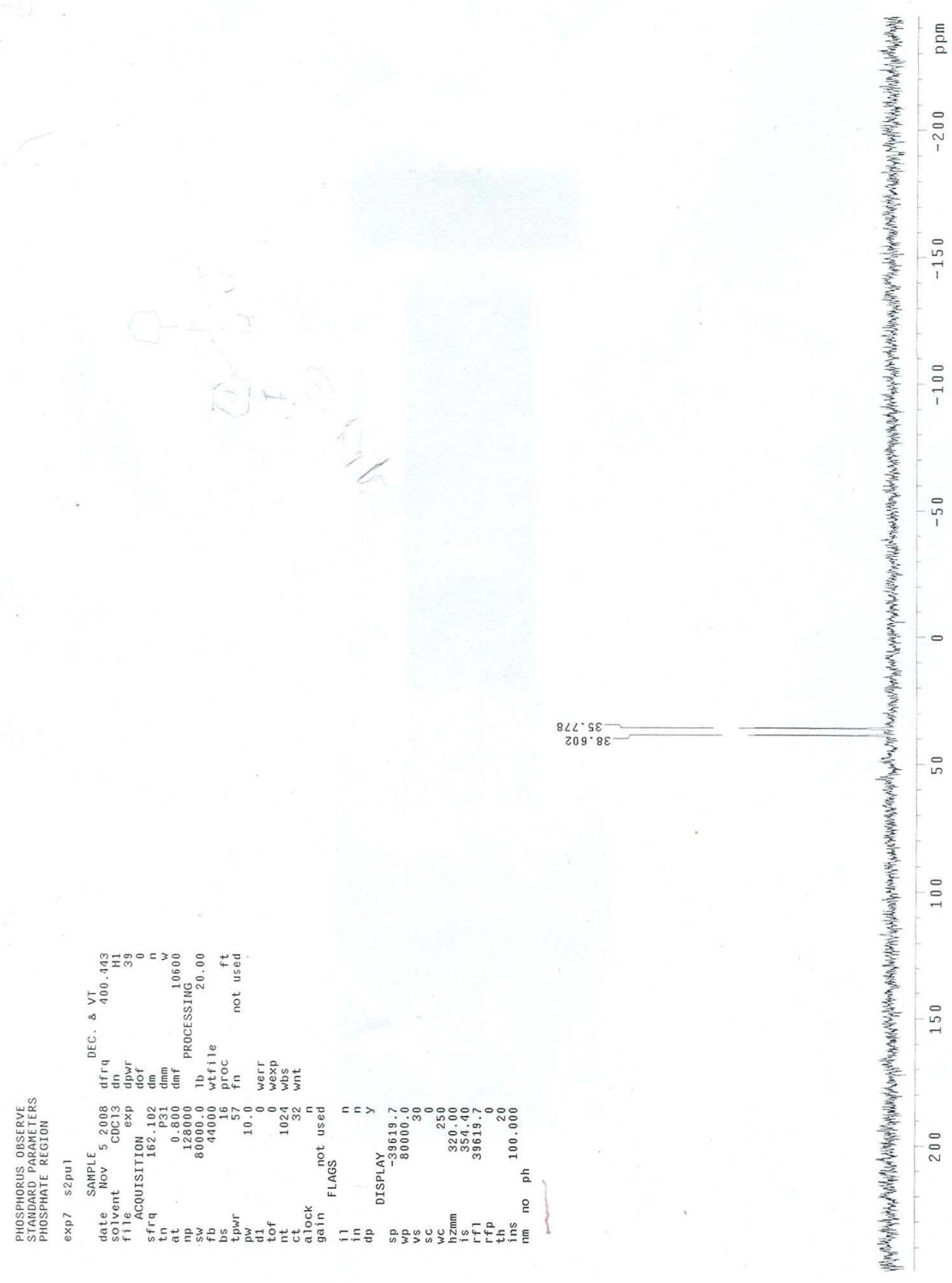

${ }^{31}$ P NMR of 7cM (coupled) 


\section{中興大學研發處貴重儀器使用中心}

元素分析儀 Elementar vario EL III CHN-OS Rapid 服務報告書 NO.1

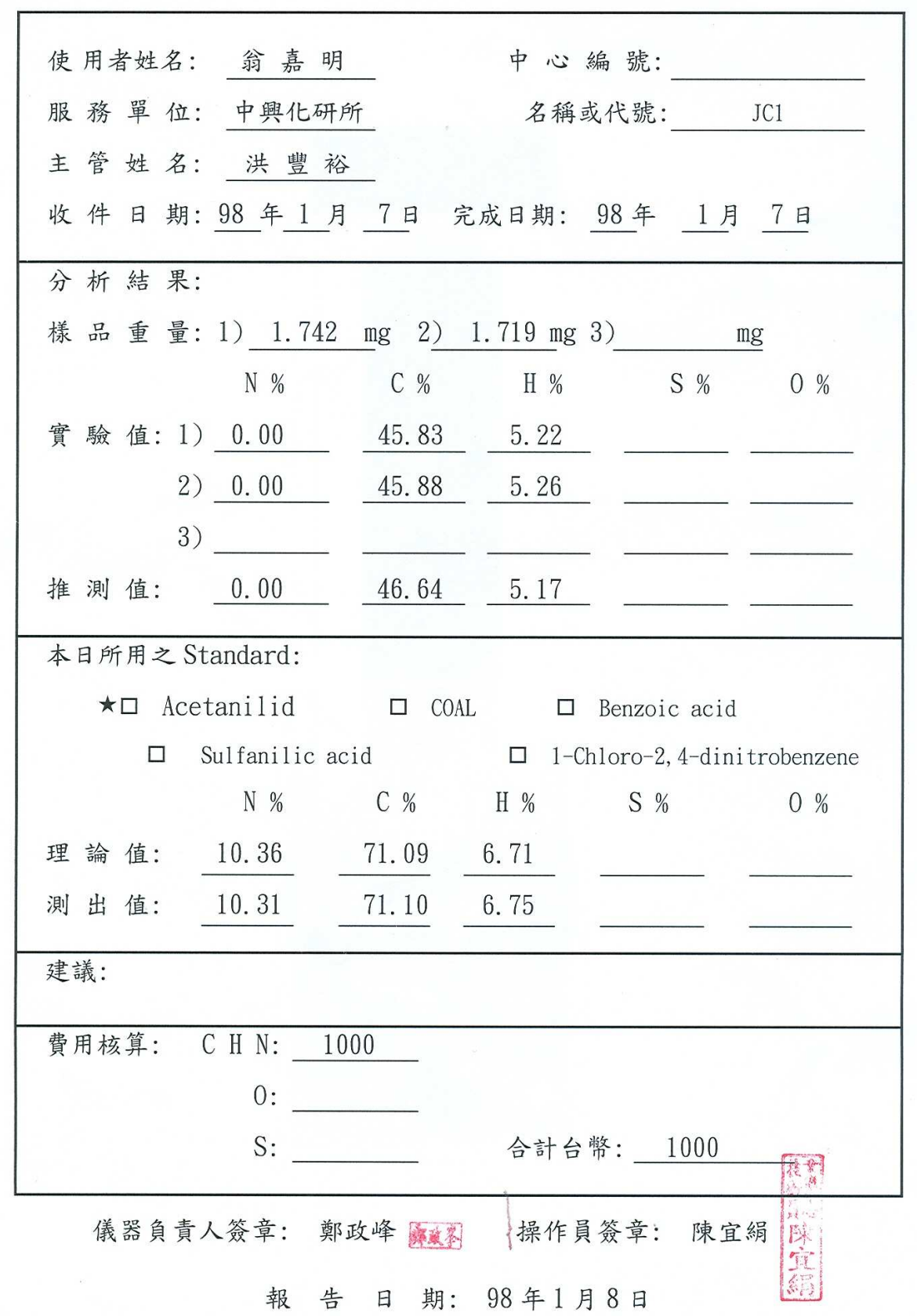

Elemental Analysis of 8:

Anal. Calcd.: C, 46.64; H,5.17.

Found: C, 45.86; H,5.24. 


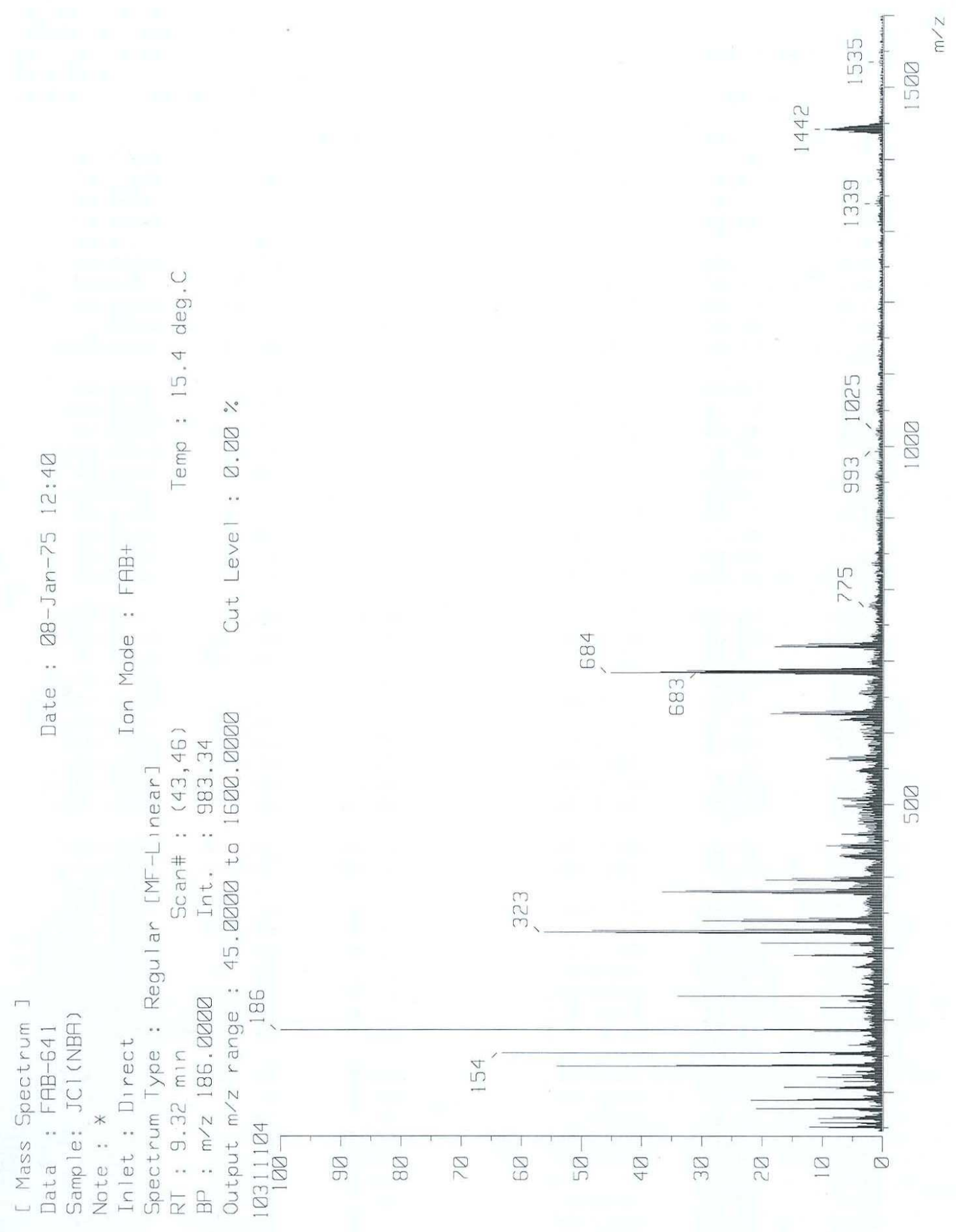

MS of 8 

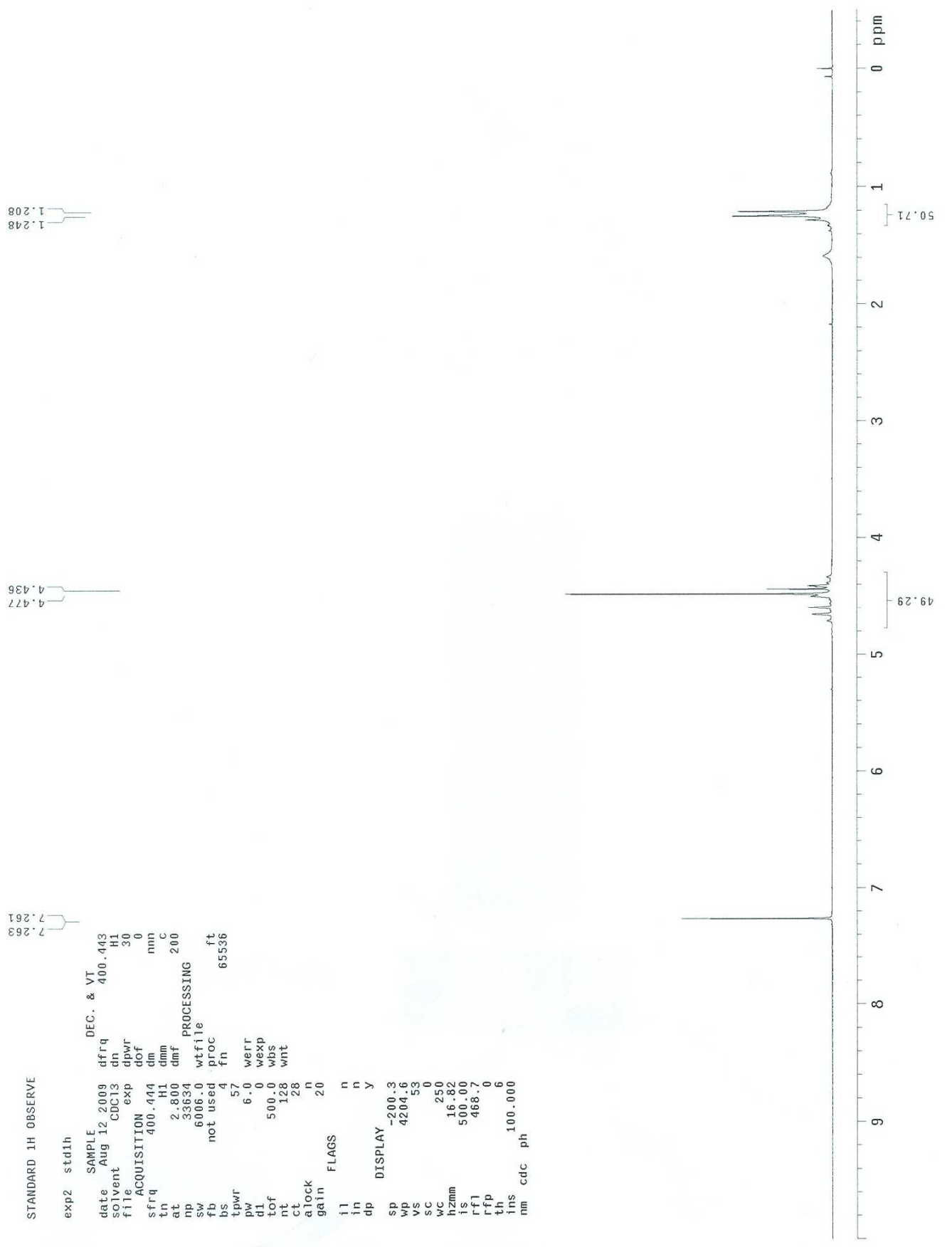

${ }^{1}$ H NMR of 8 


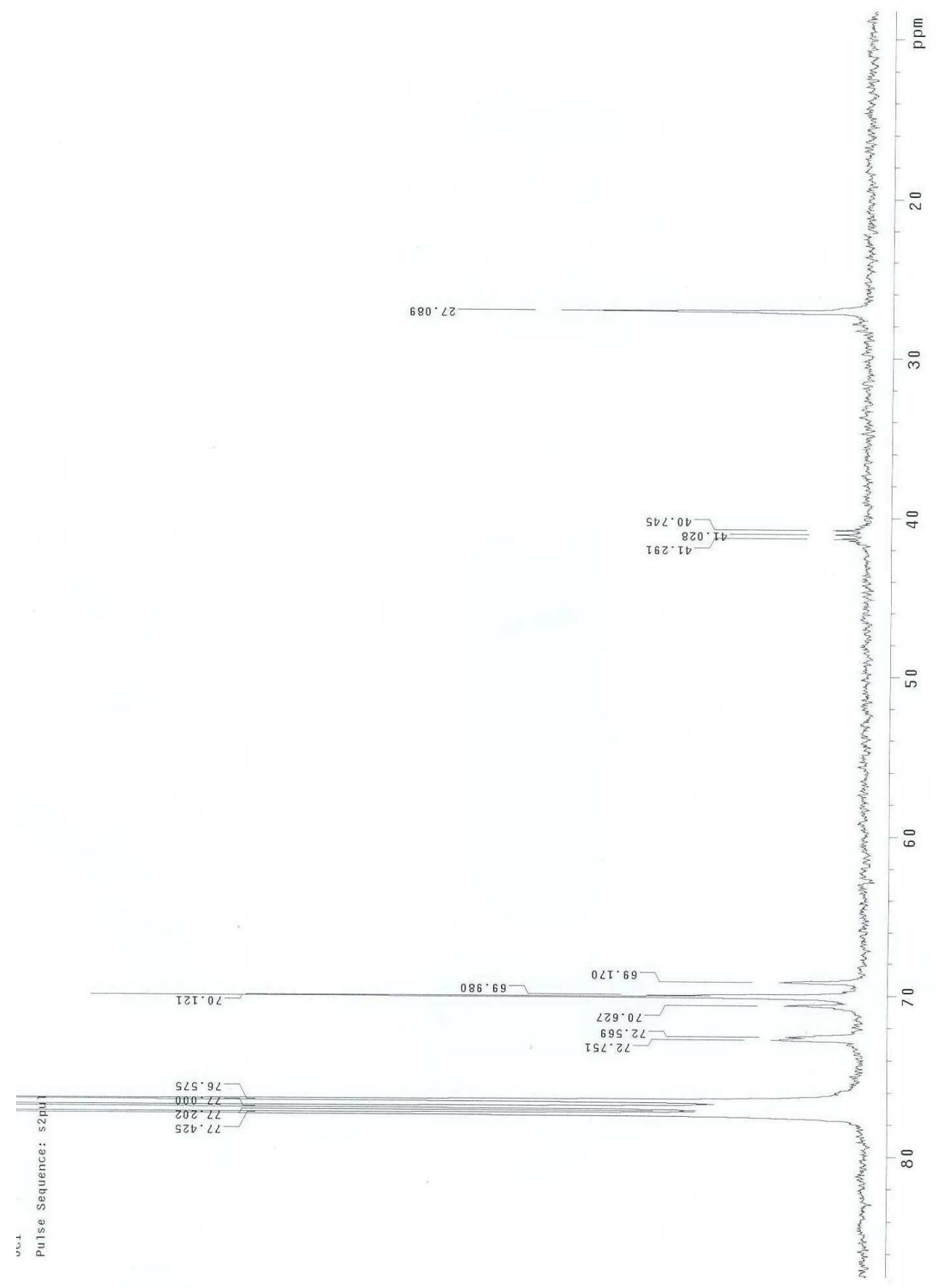

${ }^{13} \mathrm{C}$ NMR of 8 


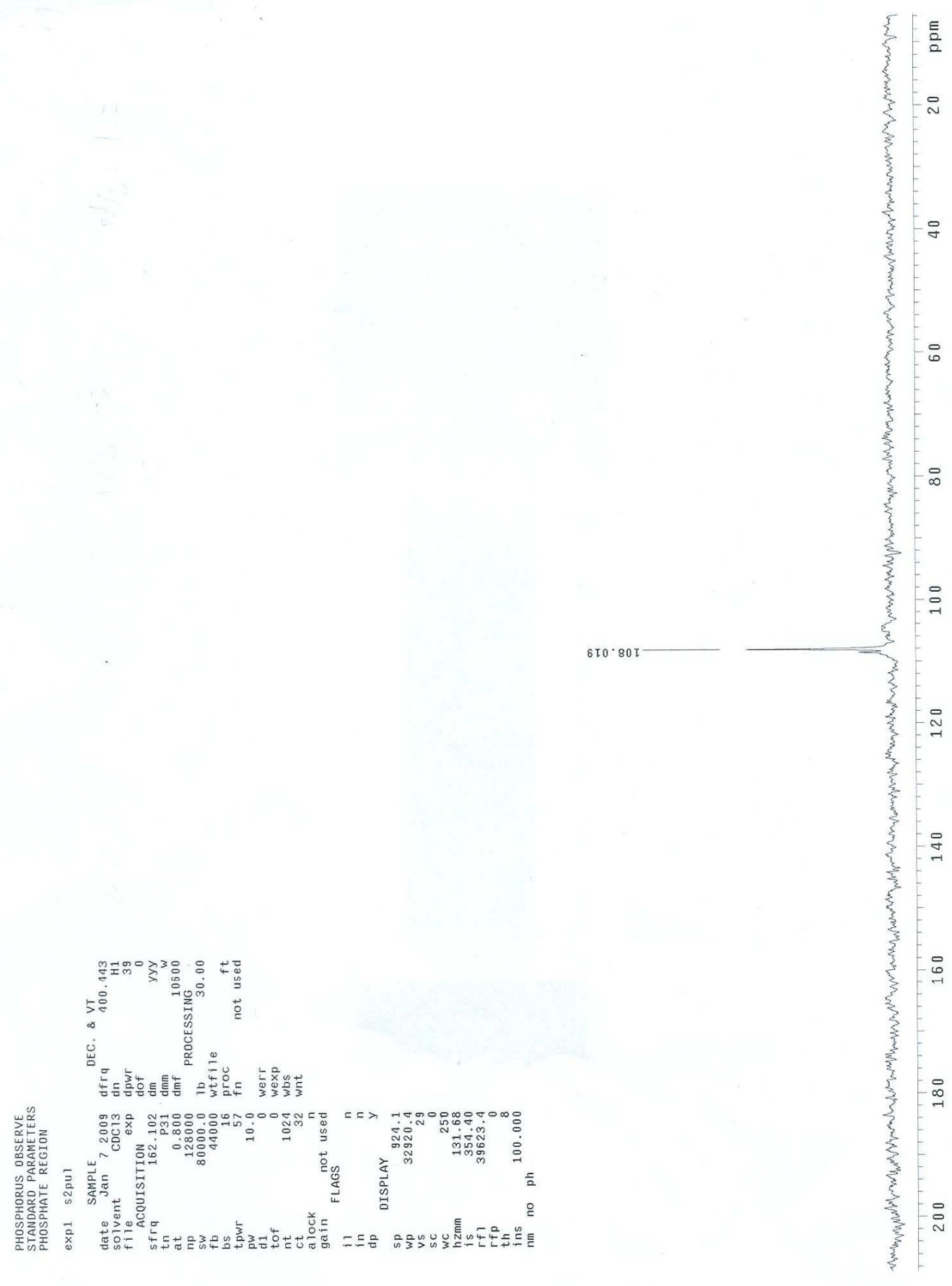

${ }^{31}$ P NMR of 8 


\section{中興大學研發處貴重儀器使用中心}

元素分析儀 Elementar vario EL III CHN-OS Rapid 服務報告書 N0.2

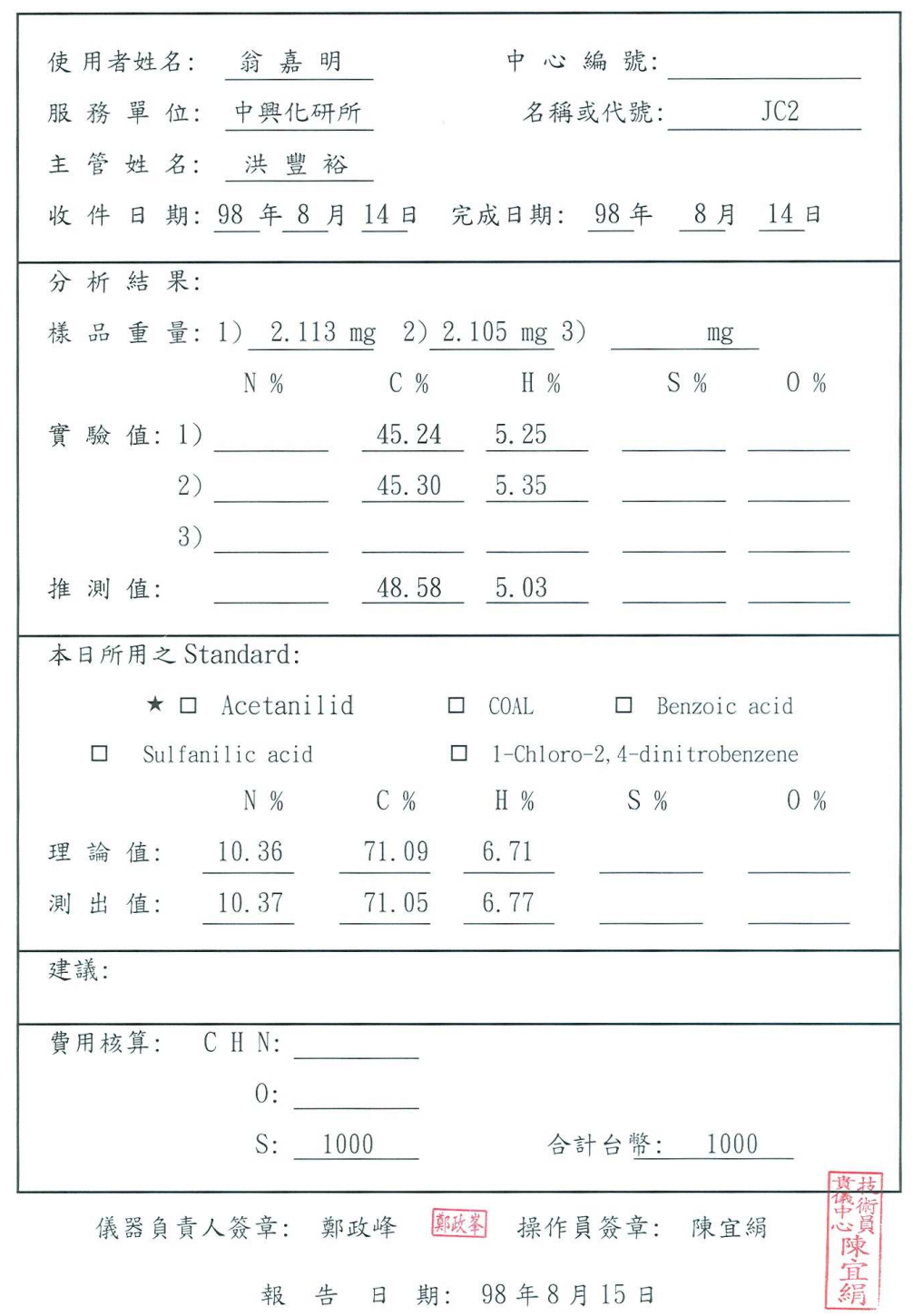

Elemental Analysis of 9:

Anal. Calcd.: C, 48.58; H, 5.03.

Found: C, 45.27; H, 5.30. 


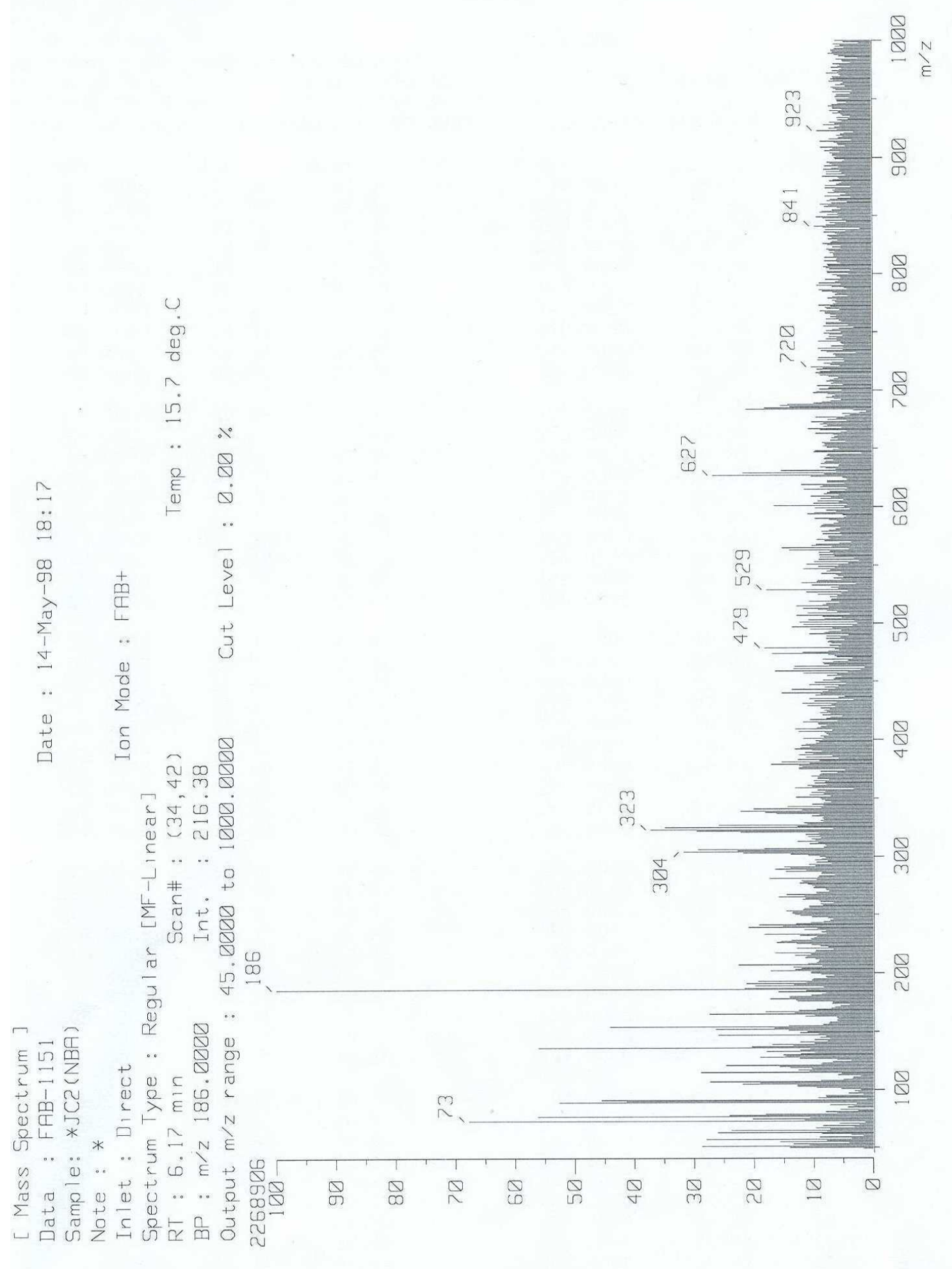

MS of 9 


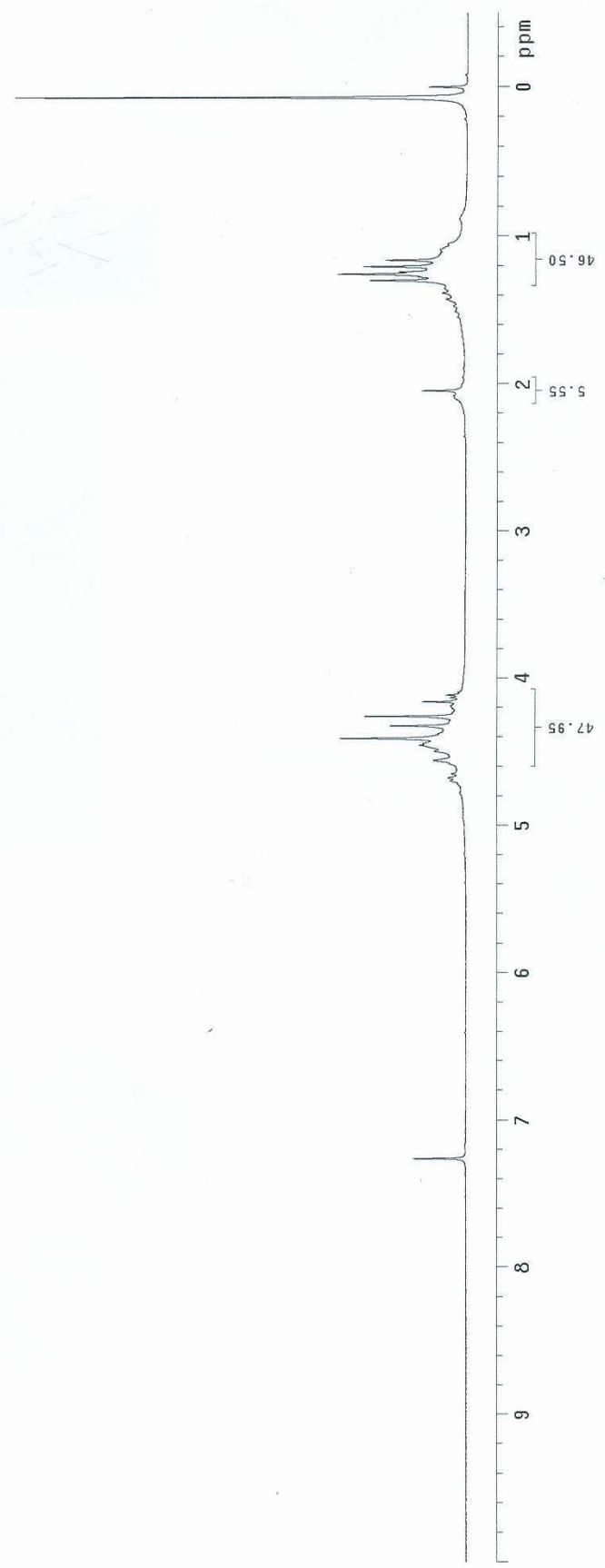

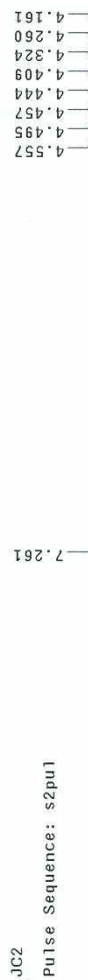

ำ $202 \cdot 1-$

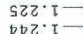

(1)

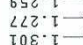

$900^{\circ} 2$

${ }^{1} \mathrm{H}$ NMR of 9

$-40-$ 


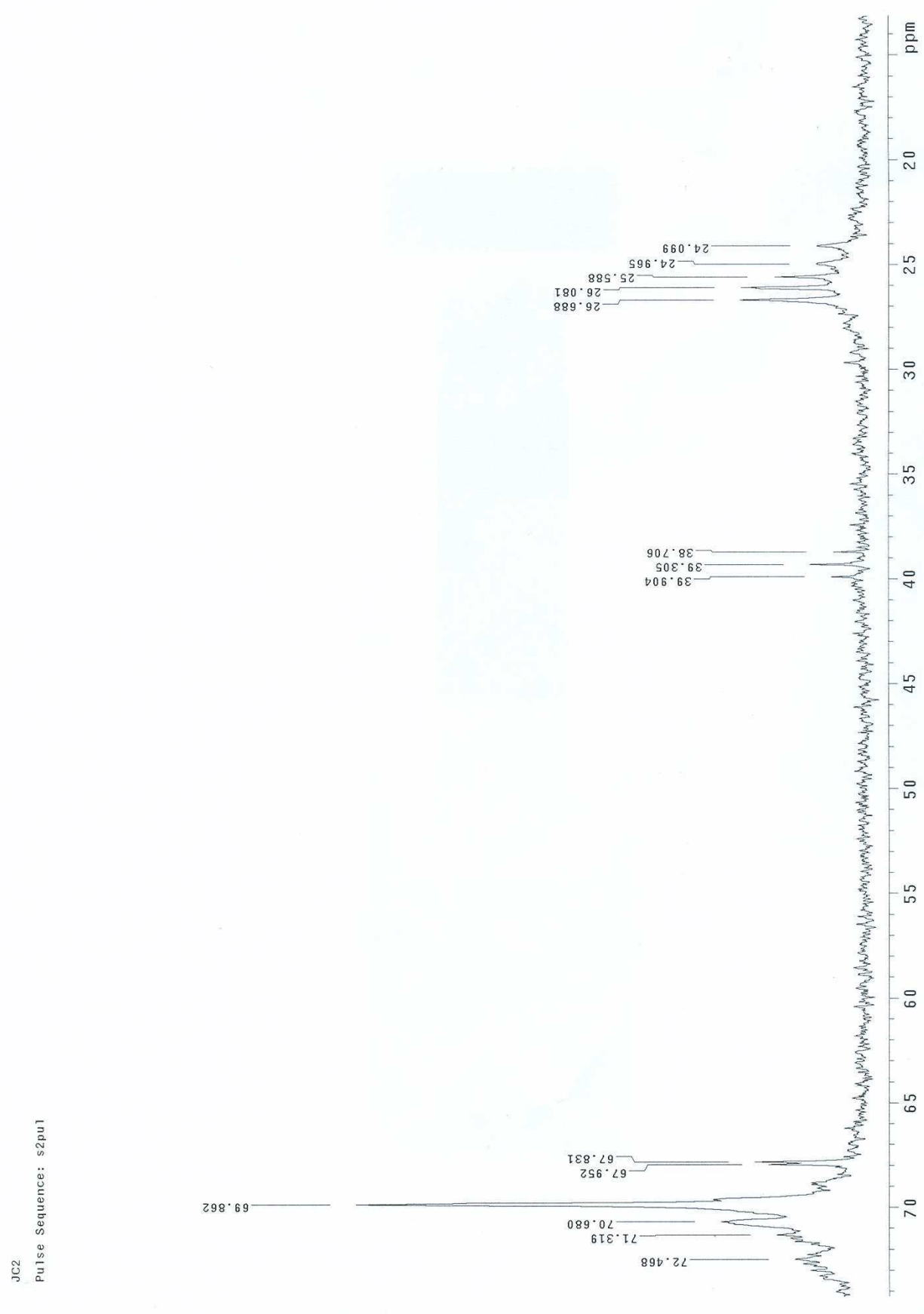

${ }^{13} \mathrm{C}$ NMR of 9 


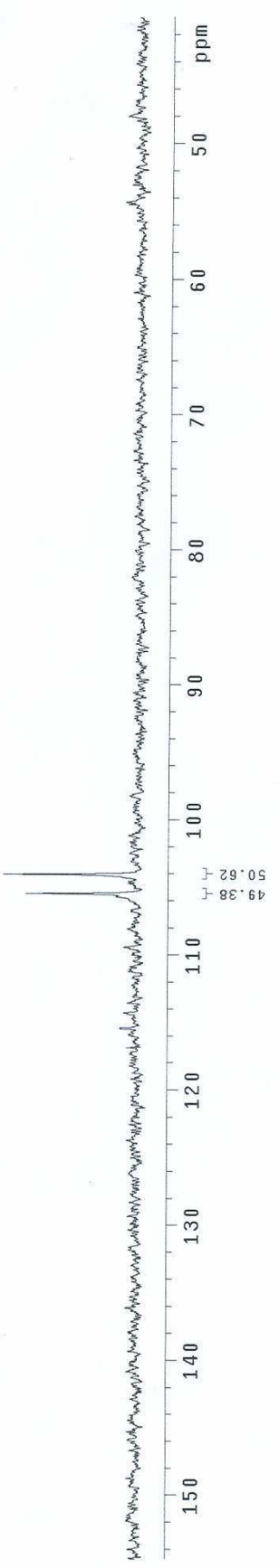

${ }^{31}$ P NMR of 9 